Aus der Klinik für Hals-Nasen-Ohrenheilkunde (Prof. Dr. Dirk Beutner)

der Medizinischen Fakultät der Universität Göttingen

\title{
Onkologische und funktionelle Ergebnisse verschiedener Therapiestrategien bei Plattenepithelkarzinomen der Subglottis
}

\author{
INAUGURAL-DISSERTATION \\ zur Erlangung des Doktorgrades \\ der Zahnmedizin \\ der Medizinischen Fakultät der \\ Georg-August-Universität Göttingen
}

vorgelegt von

Mina Mohammadpour

aus

Rheda-Wiedenbrück 
Dekan:

\section{Betreuungsausschuss}

Betreuer/in

Ko-Betreuer/in:

\section{Prüfungskommission}

Referent/in

Ko-Referent/in:

Drittreferent/in:

Datum der mündlichen Prüfung:
Prof. Dr. med. W. Brück

Prof. Dr. med. F. Ihler

Prof. Dr. med. N. Feltgen

Prof. Dr. med. F. Ihler

Prof. Dr. med. N. Feltgen

Prof. Dr. med. N. Miosge 
Hiermit erkläre ich, die Dissertation mit dem Titel "Onkologische und funktionelle Ergebnisse verschiedener Therapiestrategien bei Plattenepithelkarzinomen der Subglottis" eigenständig angefertigt und keine anderen als die von mir angegebenen Quellen und Hilfsmittel verwendet zu haben.

Göttingen, den................

(Unterschrift) 
Teile der vorliegenden Promotionsschrift wurden bereits auf einer wissenschaftlichen Konferenz in Form eines kommentierten Posters vorgestellt. Der Abstract ist veröffentlicht, das Poster online verfügbar:

Weiss B, Mohammadpour M, Canis M, Jakob M, Ihler F (2018): Transorale Lasermikrochirurgie für primär subglottische Karzinome - eine therapeutische Möglichkeit? Laryngo-Rhino-Otol $\underline{\text { 97, }} 140$

Abstract- und Posterband - 89. Jahresversammlung der Deutschen Gesellschaft für HNOHeilkunde, Kopf- und Hals-Chirurgie e.V., Bonn - Forschung heute - Zukunft morgen 


\section{Inhaltsverzeichnis}

$\begin{array}{ll}\text { Abbildungsverzeichnis } & \text { III }\end{array}$

Tabellenverzeichnis IV

$\begin{array}{lll}\text { Abkürzungsverzeichnis } & \text { V }\end{array}$

1 Einleitung 1

$\begin{array}{lll}1.1 & \text { Einführung } & 1\end{array}$

$\begin{array}{lll}1.2 & \text { Der Larynx }\end{array}$

1.2.1 Anatomie 2

1.2.2 Funktion 3

1.3 Larynxkarzinome 4

1.3.1 Epidemiologie und Ätiologie 4

1.3.2 Symptome und Diagnostik 5

1.3.3 TNM-Klassifikation und Stadiengruppierung 6

1.3.4 Prognose 9

$\begin{array}{ll}1.4 \text { Therapiemöglichkeiten } & 10\end{array}$

$\begin{array}{lll}\text { 1.4.1 Transorale Lasermikrochirurgie } & 10\end{array}$

1.4.1.1 Vorteile der TLM 11

1.4.1.2 Nachteile der TLM 12

1.4.2 Primäre Radio(chemo)therapie 12

1.4.3 Laryngektomie 13

1.4.4 Adjuvante Radio(chemo)therapie 15

1.4.5 Neck dissection 15

1.4.6 Tumornachsorge 16

$\begin{array}{ll}1.5 \text { Ziel der Studie } & 17\end{array}$

2 Patienten und Methode $\quad 18$

$\begin{array}{ll}2.1 \text { Ethik } & 18\end{array}$

$\begin{array}{ll}2.2 \text { Datenerhebung } & 18\end{array}$

$\begin{array}{lc}2.3 \text { Patientenkollektiv } & 18\end{array}$

2.3.1 Einschlusskriterien 19

2.3.2 Ausschlusskriterien 19

2.4 Staging und Nachbeobachtungszeitraum 20

$\begin{array}{ll}2.5 \text { Statistik } & 20\end{array}$

3 Ergebnisse $\quad 22$

3.1 Alters- und Geschlechtsverteilung $\quad 22$

$\begin{array}{ll}3.2 \text { Nachbeobachtung } & 22\end{array}$ 
3.3 Verteilung der TNM-Kategorien $\quad 22$

3.4 Verteilung der UICC-Stadien $\quad 24$

3.5 Therapie des Primärtumors $\quad 25$

3.5.1 Adjuvante Radio(chemo)therapie $\quad 29$

3.5.2 Neck dissection 29

3.6 Zweittumoren und relevante Nebendiagnosen 30

3.7 Onkologische Ergebnisse $\quad 30$

3.7.1 Onkologische Ergebnisse zum Zeitpunkt der letzten Tumornachsorge 30

3.7.2 Therapieversager 31

3.8 Überlebenszeitanalysen 33

3.8.1 Lokale Tumorkontrolle 33

3.8.2 Fünf-Jahres-Gesamtüberleben $\quad 35$

3.8.3 Fünf-Jahres krankheitsspezifisches Überleben 37

3.8.4 Rezidivfreies Überleben 39

3.9 Perioperatives Management, Komplikationen und funktionelle 41

Ergebnisse

3.9.1 Perioperatives Management 41

3.9.2 Komplikationen 41

3.9.3 Funktionelle Ergebnisse $\quad 42$

4 Diskussion 43

4.1 Limitationen der Studie $\quad 52$

4.2 Stärken der Studie $\quad 52$

5 Zusammenfassung und Schlussfolgerung 53

6 Literaturverzeichnis $\quad 55$ 


\section{Abbildungsverzeichnis}

Abbildung 1: Anatomie des Larynx 3

Abbildung 2: Therapiestrategien des Primärtumors 25

Abbildung 3: Therapiestrategie bei T2-Tumoren $\quad 27$

Abbildung 4: Therapiestrategie bei T3-Tumoren 28

Abbildung 5: Therapiestrategie bei T4-Tumoren 28

Abbildung 6: Kaplan-Meier-Schätzung für die lokale Kontrollrate des Gesamt- 33 kollektivs (alle Therapiestrategien)

Abbildung 7: Kaplan-Meier-Schätzung für die lokale Kontrollrate der primär lasermikrochirurgisch behandelten Patienten

Abbildung 8: Kaplan-Meier-Schätzung für das Gesamtüberleben des Gesamtkollektivs (alle Therapiestrategien)

Abbildung 9: Kaplan-Meier-Schätzung für das Gesamtüberleben der primär lasermikrochirurgisch behandelten Patienten

Abbildung 10: Kaplan-Meier-Schätzung für das krankheitsspezifische Überleben des Gesamtkollektivs (alle Therapiestrategien)

Abbildung 11: Kaplan-Meier-Schätzung für das krankheitsspezifische Überleben der primär lasermikrochirurgisch behandelten Patienten

Abbildung 12: Kaplan-Meier-Schätzung für das rezidivfreie Überleben des Gesamtkollektivs (alle Therapiestrategien)

Abbildung 13: Kaplan-Meier-Schätzung für das rezidivfreie Überleben der primär lasermikrochirurgisch behandelten Patienten 


\section{Tabellenverzeichnis}

$\begin{array}{ll}\text { Tabelle 1: } \text { T-Kategorie } & 7\end{array}$

Tabelle 2: N-Kategorie $\quad 8$

Tabelle 3: M-Kategorie $\quad 8$

Tabelle 4: Stadieneinteilung subglottischer Tumore nach UICC 9

Tabelle 5: Primärtumor - Verteilung der T-Kategorien 23

Tabelle 6: Halslymphknotenmetastasierung - Verteilung der N-Kategorien 23

Tabelle 7: Primärtumor - Kombination der T- und N-Kategorien 23

Tabelle 8: Verteilung der UICC-Stadien $\quad 24$

Tabelle 9: Therapie des Primärtumors in Abhängigkeit von der T-Kategorie 26

Tabelle 10: Therapie des Primärtumors in Abhängigkeit vom UICC-Stadium 27

Tabelle 11: Onkologische Ergebnisse zum Zeitpunkt der letzten Tumornachsorge 31

Tabelle 12: Häufigkeit und Art des ersten Rezidivs 32

Tabelle 13: Häufigkeit und Art des zweiten Rezidivs 32

Tabelle 14: Zusammenfassung der Publikationen zur Therapie subglottischer 49 Plattenepithelkarzinome 


\section{Abkürzungsverzeichnis}

DNA

Gy

LE

M

$\mathrm{N}$

ND

OP

part. LE

PEG

prim. RCT

RCT

RT

$\mathrm{T}$

Tis

TLM

UICC
Desoxyribonukleinsäure

Gray

Laryngektomie

Fehlen oder Vorhandensein von Fernmetastasen

Fehlen oder Vorhandensein und Ausdehnung von regionären

Lymphknotenmetastasen

Neck dissection

Operation

partielle Laryngektomie

Perkutane endoskopische Gastrostomie

primäre Radiochemotherapie

Radiochemotherapie

Radiotherapie

Ausdehnung des Primärtumors

Carcinoma in situ

Transorale Lasermikrochirurgie

The Union for International Cancer Control 


\section{Einleitung}

\subsection{Einführung}

In der Bundesrepublik Deutschland erkranken circa 3500 Menschen pro Jahr an einem Larynxkarzinom. Den häufigsten histologischen Typ stellt dabei das Plattenepithelkarzinom dar (ZfKD 2016). Bei nur etwa $5 \%$ der Larynxkarzinome ist der Tumor in der Subglottis lokalisiert (Schuler et al. 2009), folglich handelt es sich bei primär subglottischen Plattenepithelkarzinomen um eine seltene Erkrankung (Ferlito und Rinaldo 2000). Die Entstehung eines Larynxkarzinoms wird begünstigt durch Alkohol- und Nikotinkonsum, außerdem ist die genetische Disposition von Bedeutung. Prognostisch ungünstig ist bei den subglottischen Karzinomen, dass sie in frühen Tumorstadien asymptomatisch und daher unerkannt bleiben. Zum Zeitpunkt der Diagnose liegen meist schon fortgeschrittene Tumoren vor (Zimmermann und Matthias 2009). Aufgrund des oft fortgeschrittenen Tumorstadiums zum Zeitpunkt der Diagnose ist die Prognose subglottischer Malignome schlecht (Dahm et al. 1998).

Zu den Therapiemöglichkeiten subglottischer Tumoren zählen neben der konventionellen offen chirurgischen Larynxteilresektion oder der nicht organerhaltenden Laryngektomie auch die primäre Radio(chemo)therapie.

In den 70er Jahren wurde in den USA erstmals die minimalinvasive transorale Lasermikrochirurgie eingesetzt, um benigne laryngeale Läsionen zu therapieren (Strong und Jako 1972). Professor Steiner aus Göttingen behandelte erstmalig Malignome des Larynx mittels transoraler Lasermikrochirurgie und erweiterte dessen Einsatzbereich auf den gesamten oberen Aerodigestivtrakt (Steiner et al. 2013; Steiner 1988).

Das Fünf-Jahres-Gesamtüberleben aller Larynxkarzinome liegt bei Männern bei $63 \%$, bei Frauen liegt es bei $61 \%$ (ZfKD 2016). Die primär subglottische Lokalisation von Larynxkarzinomen stellt dabei eine Seltenheit dar (Ferlito und Rinaldo 2000). Dem entsprechend liegen nur wenige Studien zu Therapiestrategien subglottischer Malignome und ihren Ergebnissen vor.

Ziel der vorliegenden Arbeit war es, die Behandlungsstrategien sowie onkologische und funktionelle Ergebnisse der seltenen Tumorentität subglottischer Plattenepithelkarzinome zu evaluieren. 


\subsection{Der Larynx}

\subsubsection{Anatomie}

Der Larynx liegt als Organ zwischen Pharynx und Trachea. Er besteht aus dem knorpeligen Kehlkopfskelett, den Kehlkopfbändern, -gelenken, -membranen und -muskeln. Das Kehlkopfskelett besteht aus dem Schildknorpel (Cartilago thyroidea), dem Ringknorpel (Cartilago cricoidea), dem Stellknorpel beziehungsweise Aryknorpel (Cartilago arytenoideae) und dem Kehldeckel beziehungsweise der Epiglottis (Cartilago epiglottica). Die einzelnen Bestandteile des Kehlkopfskeletts werden durch Bänder, Gelenke und Membranen miteinander, mit der Trachea und dem Zungenbein verbunden. Durch die Kehlkopfmuskulatur wird einerseits das Kehlkopfskelett gegeneinander bewegt, andererseits bestimmt sie die Stellung der Stimmbänder (Ligamentum vocale). Der Musculus vocalis hat seinen Ursprung an der Rückfläche des Schildknorpels und setzt am Processus vocalis des Stellknorpels an (Fritsch und Kühnel 2009).

Der Larynx wird in drei Etagen unterteilt: Die obere Etage, Supraglottis (Vestibulum laryngis), erstreckt sich vom Kehlkopfeingang (Epiglottis und den beiden Plicae aryepiglotticae) bis zu den Taschenfalten (Plicae vestibulares). Die mittlere Etage, die Glottis (Cavitas laryngis intermedia), ist am kleinsten und beginnt an den Taschenfalten. Sie endet unter den Stimmlippen (Plicae vocales). Die untere Etage, die Subglottis (Cavitas infraglottica), reicht von den Stimmlippen bis zum unteren Rand des Ringknorpels (Fritsch und Kühnel 2009). Während in der Literatur Einigkeit über die inferiore Begrenzung der Subglottis herrscht, gehen die Meinungen bezüglich der superioren Begrenzung jedoch auseinander (Garas und McGuirt 2006; Ferlito und Rinaldo 2000). Während einerseits die obere Begrenzung des Conus elasticus als superiore Begrenzung der Subglottis bezeichnet wird, heißt es andererseits, die inferiore Begrenzung der Stimmlippen oder bis zu $1 \mathrm{~cm}$ darunter bilde die superiore Begrenzung der Subglottis (Garas und McGuirt 2006; Ferlito und Rinaldo 2000).

Histologisch betrachtet ist der Larynx von mehrschichtig respiratorischem Flimmerepithel überzogen, einzig die Plica vocalis ist von einem mehrschichtig unverhornten Plattenepithel bedeckt (Fritsch und Kühnel 2009). 


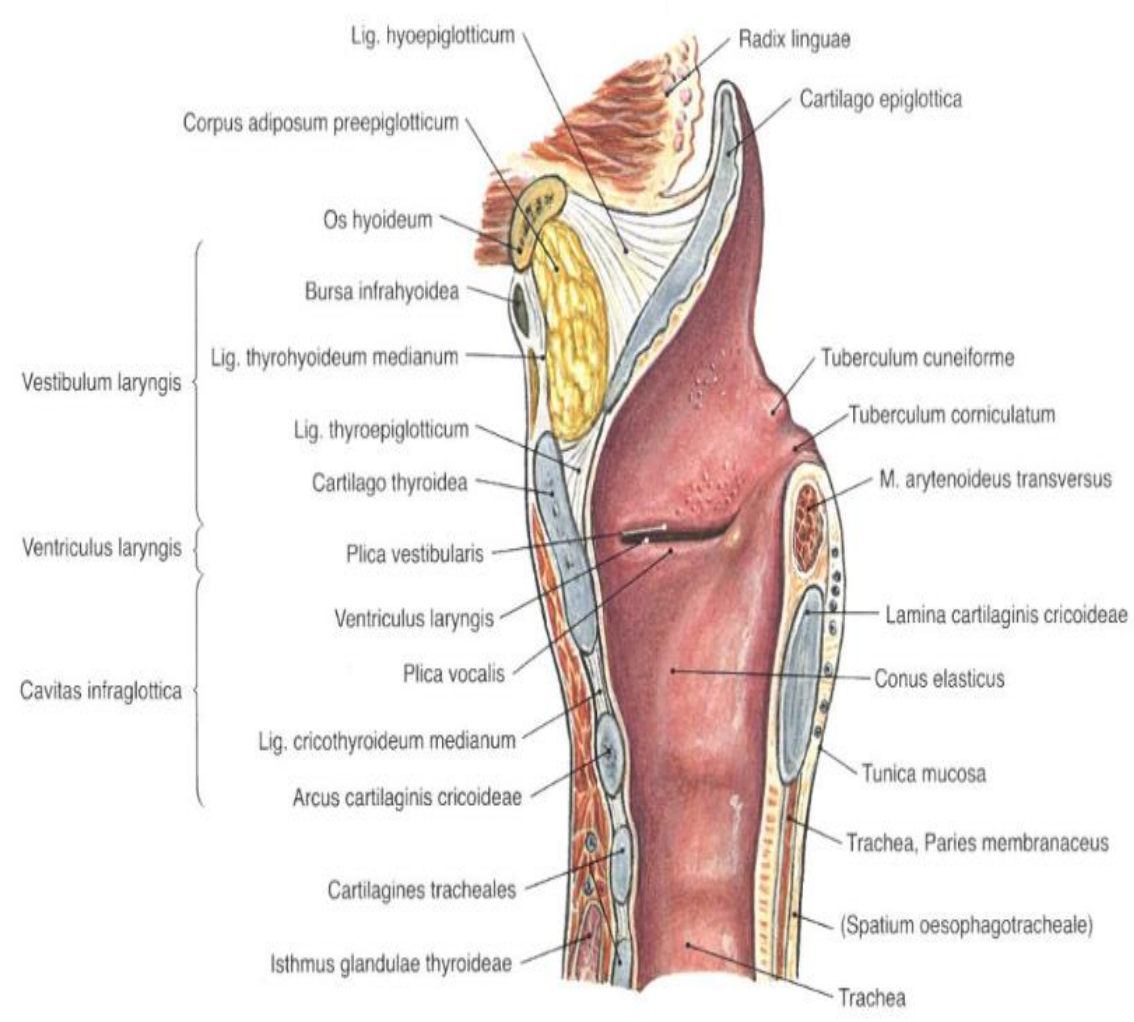

Abbildung 1: Anatomie des Larynx (Sobotta 2017)

Die Verwendung der Abbildung erfolgt mit freundlicher Genehmigung des Elsevier-Verlags.

\subsubsection{Funktion}

Der Larynx erfüllt drei wichtige Funktionen: Er hat eine Schutzfunktion und dient zur Phonation und als Atemweg.

Er schützt die weiter aboral liegenden Luftwege vor Nahrung und Speichel, ähnlich einem Ventil, indem er beim Schluckakt hochsteigt, sodass die Epiglottis den Durchgang zum Larynx verhindert. Ferner legen sich die Stimmlippen beim Schlucken aneinander und vermeiden so zusätzlich die Aspiration. Sollte ein Fremdkörper in den Larynx gelangen, wird ein Hustenreflex ausgelöst, um die unteren Atemwege $\mathrm{zu}$ reinigen (Lenarz und Boenninghaus 2012).

Zudem ermöglicht der Kehlkopf die Phonation, indem er zu Beginn der Tonerzeugung die Stimmritze schließt und die Stimmbänder anspannt. Als nächstes öffnet ein exspiratorischer Luftstrom die Stimmritze und führt zur Schwingung der Stimmfalten, was zur Entstehung von Schallwellen führt. Diese Schallwellen werden in ihrer Lautstärke und Tonhöhe durch 
die Stärke des Luftstroms beziehungsweise der Schwingungsfrequenz modifiziert. Weitere, auf die Frequenz Einfluss ausübende Faktoren sind die Länge, Dicke und Spannung der Stimmbänder (Fritsch und Kühnel 2009).

Auch ermöglicht der Larynx dem menschlichen Organismus die Atmung, indem er die engste Stelle des Organs durch die Respirationsstellung der Stimmbänder dem Luftstrom passierbar macht (Lenarz und Boenninghaus 2012).

Zudem ist durch einen Glottisschluss die Bauchpresse möglich. Der hohe intraabdominelle Druck ermöglicht die Darmentleerung, das Erbrechen sowie das Austreiben eines Kindes während des Geburtsvorgangs (Moll 2006).

\subsection{Larynxkarzinome}

\subsubsection{Epidemiologie und Ätiologie}

Im Jahr 2013 erkranken 252.600 Männer und 229.900 Frauen in Deutschland an bösartigen Neubildungen. Die Anzahl an Neuerkrankungen bei Larynxkarzinomen liegt im selben Jahr bei Männern bei 2.990, während sie bei Frauen 530 zählt (ZfKD 2016). Männer leiden rund fünf Mal häufiger an einem Larynxkarzinom als Frauen (Zimmermann und Matthias 2009; Steuer et al. 2017). Der Altersgipfel liegt zwischen dem 55. und 65. Lebensjahr (Iro und Waldfahrer 2008). Das Larynxkarzinom macht mit 20,9 \% aller Kopf-Hals-Malignome den häufigsten Tumor des oberen Aerodigestivtrakts aus (Hoffman et al. 1998). Nur etwa $5 \%$ aller Kehlkopftumore sind in der Subglottis lokalisiert, häufiger sind die Glottis (60\%) und die Supraglottis (35\%) betroffen (Schuler et al. 2009; Levy et al. 2014). Zum Zeitpunkt der Diagnose besteht verglichen mit glottischen Karzinomen häufig eine fortgeschrittene TKategorie (Shin und Troung 2015).

Der Entstehung von Kehlkopfkrebs liegen einerseits exogene, andererseits endogene Faktoren zugrunde. Zu den exogenen Faktoren zählen Nikotinkonsum sowie Alkoholabusus (Zimmermann und Matthias 2009; Ramroth et al. 2006). Die beiden Genussmittel stellen die wichtigsten Risikofaktoren dar. Eine Kombination aus Nikotin und Alkohol erhöht die kanzerogene Wirkung im Vergleich zum alleinigen Konsum von Alkohol beziehungsweise Nikotin (Cattaruzza et al. 1996). Ferner zählen auch etliche Schadstoffe wie Quarze, Zementstaub und Teerprodukte zu möglichen exogenen Ursachen von Kehlkopfkrebs, 
ihnen hingegen wird eine geringere Bedeutung beigemessen (Zimmermann und Matthias 2009).

Von Larynxkarzinomen sind jedoch auch Patienten betroffen, die keinen exzessiven Genuss von Alkohol und Nikotin betreiben. In diesen Fällen wird eine genetische Disposition angenommen. Diese Patienten verfügen über ein geschädigtes DNA-Reparatursystem (Desoxyribonukleinsäure) im Bereich der Schleimhaut und über genetische Defekte entgiftender Enzyme. Hier liegt der Altersgipfel mit 20 bis 40 Jahren früher (Zimmermann und Matthias 2009).

\subsubsection{Symptome und Diagnostik}

Subglottische Karzinome bleiben in frühen Tumorstadien asymptomatisch, sodass sie meist erst spät diagnostiziert werden (Santoro et al. 2000). Zum Zeitpunkt der Diagnose liegt nur in seltenen Fällen ein T1- oder T2-Karzinom vor (DKG 2019). Zu den häufigsten Symptomen zählen Dyspnoe und Stridor (Ferlito und Rinaldo 2000). Erreicht das zunächst primär subglottische Malignom die Glottis und infiltriert die Stimmlippen, führt dies zum Symptom der Heiserkeit (Harrison 1971).

Bestandteil der prätherapeutischen Diagnostik eines Larynxkarzinoms ist unter anderem eine visuelle Untersuchung des Organs mittels Lupenlaryngoskopie. Frühe asymptomatische Malignome der Subglottis können dabei aufgrund der möglicherweise eingeschränkten Einsicht jedoch leicht übersehen werden (Guedea et al. 1991; Dahm et al. 1998). Darüber hinaus kann zusätzlich eine Stroboskopie erfolgen, etwa wenn die Ursache für eine begrenzte Stimmlippenbeweglichkeit unklar ist. Unverzichtbar zur Erkennung eines Lymphknotenbefalls ist die Halspalpation gefolgt von der sonographischen Untersuchung. Ebenfalls mit der Frage nach Halslymphknotenbefall, zudem aber auch zur Evaluation der Tumorausdehnung und einer möglichen Infiltration des knorpeligen Kehlkopfskeletts, ist eine Computertomographie indiziert. Des Weiteren erfolgt im Rahmen der Diagnosestellung und des Tumor-Stagings, also der prätherapeutischen Stadieneinteilung, eine Panendoskopie in Vollnarkose. Diese dient dem Ausschluss von Zweittumoren im oberen Aerodigestivtrakt und ermöglicht die Bestimmung der Tumorausdehnung (Zimmermann und Matthias 2009). Zudem wird während der Panendoskopie eine Probe des veränderten Gewebes zur histopathologischen Untersuchung und damit Diagnosestellung gewonnen. Komplettiert 
wird das prätherapeutische Staging durch eine Computertomographie des Thorax sowie eine Sonographie des Abdomens zur Ermittlung möglicher Fernmetastasen.

\subsubsection{TNM-Klassifikation und Stadiengruppierung}

Die Erkenntnis zeigt, dass die Überlebenswahrscheinlichkeit bei Patienten mit einer lokal begrenzten Tumorerkrankung günstiger ist als die einer ausgedehnteren Tumorerkrankung, welche bereits Nachbarstrukturen befallen hat. So entwickelte Pierre Denoix zwischen 1943 und 1952 die TNM-Klassifikation, indem er Malignome nach ihrer anatomischen Ausdehnung einteilt. Ein Jahr später einigt sich die Union for International Cancer Control (UICC) darauf, das TNM-System zur Klassifikation von Tumoren zu übernehmen. Das Larynxkarzinom wurde erstmalig 1958 nach dem TNM-System klassifiziert (Hermanek et al. 1992). In die TNM-Klassifikation fließen drei Bestandteile ein:

T - Ausdehnung des Primärtumors

$\mathrm{N}$ - Fehlen oder Vorhandensein und Ausdehnung von regionären Lymphknotenmetastasen $\mathrm{M}$ - Fehlen oder Vorhandensein von Fernmetastasen

Jeder Tumor bedarf einer mikroskopischen Diagnosestellung. Unabhängig davon werden zwei Klassifikationen unterschieden: Die klinische, also prätherapeutische beziehungsweise präoperative Klassifikation beinhaltet das Ergebnis der Staging-Untersuchungen. Diese wird als cTNM gekennzeichnet. Die pathologische oder postoperative Klassifikation wird nach histopathologischer Untersuchung des operativ entnommenen Gewebes durch den Patholgen festgelegt. In diesem Fall erfolgt die Kennzeichnung durch pTNM. Die Klassifikation von Rezidiven erfolgt durch rTNM (Hermanek et al. 1992).

Das TNM-System ist ein unverzichtbarer Bestandteil der onkologischen Behandlung, da es mit einer international anerkannten und einheitlichen Klassifikation etliche Vorteile und Möglichkeiten bietet. Es ermöglicht die Erstellung eines individuellen, stadiengerechten Behandlungsplans und vereinfacht den Informationsaustausch zwischen Kliniken. Ferner bietet es Hinweise auf die Prognose und ermöglicht die Auswertung von Behandlungsergebnissen. Letztlich können durch das TNM-System Tumorerkrankungen und Behandlungskonzepte erforscht werden (Hermanek et al. 1992).

In der vorliegenden Arbeit wurden die Tumoren der Subglottis unter Berücksichtigung der siebten Edition der UICC-Staging-Klassifikation ausgewertet (Sobin und Compton 2010), siehe Tabellen 1 bis 3 . 
Tabelle 1: T-Kategorie (Sobin und Compton 2010)

\begin{tabular}{|c|l|}
\hline TX & Primärtumor kann nicht beurteilt werden \\
\hline T0 & Kein Anhalt für Primärtumor \\
\hline Tis & Carcinoma in situ \\
\hline T1 & Tumor auf die Subglottis begrenzt \\
\hline T2 & $\begin{array}{l}\text { Tumor breitet sich auf ein oder beide Stimmbänder aus, diese mit } \\
\text { normaler oder eingeschränkter Beweglichkeit }\end{array}$ \\
\hline T3 & $\begin{array}{l}\text { Tumor auf den Larynx begrenzt, mit Stimmlippenfixation } \\
\text { außerhalb des Kehlkopfes aus, z.B. Trachea, Weichteile des Halses } \\
\text { eingeschlossen äußere Muskulatur der Zunge (Musculus genioglossus, } \\
\text { Musculus byoglossus, Musculuspalatoglossus und Musculus styloglossus), gerade } \\
\text { Halsmuskulatur, Schilddrüse, Ösophagus }\end{array}$ \\
\hline T4b & $\begin{array}{l}\text { Tumor infiltriert den Prävertebralraum, mediastinale Strukturen oder } \\
\text { umschließt die Arteria carotis interna }\end{array}$ \\
\hline
\end{tabular}


Tabelle 2: N-Kategorie (Sobin und Compton 2010)

\begin{tabular}{|r|l|}
\hline NX & Regionäre Lymphknoten können nicht beurteilt werden \\
\hline N0 & Keine regionären Lymphknotenmetastasen \\
\hline N1 & $\begin{array}{l}\text { Metastase(n) in solitärem ipsilateralem Lymphknoten, } \leq 3 \mathrm{~cm} \text { in } \\
\text { größter Ausdehnung }\end{array}$ \\
\hline N2 & Metastase(n) wie nachfolgend beschrieben: \\
\hline N2a & $\begin{array}{l}\text { Metastase(n) in solitärem ipsilateralem Lymphknoten, 3 }-6 \mathrm{~cm} \text { in } \\
\text { seiner Ausdehnung }\end{array}$ \\
\hline N2b & $\begin{array}{l}\text { Metastasen in multiplen ipsilateralen Lymphknoten, keine größer als } \\
\text { 6 cm in größter Ausdehnung }\end{array}$ \\
\hline N2c & $\begin{array}{l}\text { Metastasen in bilateralen oder kontralateralen Lymphknoten, keine } \\
\text { größer als 6 cm in ihrer größten Ausdehnung }\end{array}$ \\
\hline N3 & Metastase(n) in Lymphknoten, > 6 cm in größter Ausdehnung \\
\hline
\end{tabular}

Tabelle 3: M-Kategorie (Sobin und Compton 2010)

\begin{tabular}{|l|l|}
\hline MX & Das Vorliegen von Fernmetastasen kann nicht beurteilt werden \\
\hline M0 & Keine Fernmetastasen \\
\hline M1 & Fernmetastasen \\
\hline
\end{tabular}

Die TNM-Klassifikation wird in der Stadiengruppierung der UICC wie in Tabelle 4 dargestellt zusammengefasst. 
Tabelle 4: Stadieneinteilung subglottischer Tumore nach UICC (Sobin und Compton 2010)

\begin{tabular}{|c|c|c|c|}
\hline Stadium & T-Kategorie & N-Kategorie & M-Kategorie \\
\hline 0 & Tis & N0 & M0 \\
\hline I & T1 & N0 & M0 \\
\hline II & T2 & N0 & M0 \\
\hline III & T1, T2 & N0, N1 & M0 \\
\hline IVA & T1, T2, T3 & N2 & M0 \\
\hline Ty & T4a & T4b N1, N2 & M0 \\
\hline IVB & Jedes T & Jedes N & M0 \\
\hline IVC & Jedes T & Jedes N & M1 \\
\hline
\end{tabular}

Inzwischen wurde die achte Edition der TNM-Klassifikation aus dem Jahr 2016 verabschiedet. Sie diente jedoch nicht als Grundlage zur Auswertung der zwischen 1992 und 2013 in der Universitätsmedizin Göttingen behandelten Patienten.

\subsubsection{Prognose}

Subglottische Malignome sind in frühen Tumorstadien asymptomatisch und werden häufig erst spät diagnostiziert (Zimmermann und Matthias 2009). Sie infiltrieren das Knorpelgewebe rascher, da sich zwischen der Mukosa und dem Ringknorpel keine Muskelschicht befindet (Guedea et al. 1991; Cassidy et al. 2012). Metastasen in regionäre Lymphknoten treten in frühen Tumorstadien, trotz der starken lymphatischen Drainage der Subglottis, selten auf. In fortgeschrittenen Stadien sind sie jedoch zahlreicher zu beobachten (Shaha und Shah 1982). Aufgrund des späten Diagnosezeitpunktes gegenüber Malignomen anderer Larynxbereiche ist die Überlebenswahrscheinlichkeit subglottischer Karzinome laut Dahm et al. (1998) schlecht. Stimmlippenkarzinome hingegen machen sich schon früh durch 
Heiserkeit bemerkbar und sind deshalb prognostisch günstiger (Lenarz und Boenninghaus 2012; Ferlito und Rinaldo 2000).

\subsection{Therapiemöglichkeiten}

\subsubsection{Transorale Lasermikrochirurgie}

Bis zur Einführung der transoralen Lasermikrochirurgie (TLM) galten Laryngektomien, Radio(chemo)therapien oder offen chirurgische Eingriffe als Therapiestandards zur Behandlung von Larynxkarzinomen (Genden et al. 2007). Transorale Eingriffe im Kehlkopf erfolgten mithilfe des $\mathrm{CO}_{2}$-Lasers (Kohlenstoffdioxid-Laser) erstmalig in den USA. Anfang der 1970er Jahre wurden sie von Jako und Strong zur Behandlung benigner laryngealer Läsionen durchgeführt (Strong und Jako 1972; Dellian 2009). Für die minimalinvasiven Eingriffe koppelten sie den Laser an ein Laryngoskop (Rudert und Werner 2011). Ende der 1970er Jahre etablierte Professor Steiner die Methode an der Universitätsmedizin Göttingen (Dellian 2009) und erweiterte den Indikationsbereich auch auf bösartige Erkrankungen und auf Tumoren des gesamten oberen Aerodigestivtrakts (Steiner 1988; Steiner und Ambrosch 2000). Prämisse für die Anwendung des Lasers sind eine gute endoskopische Einstellbarkeit des tumorösen Gewebes und deutlich sichtbare Tumorgrenzen (Steiner et al. 2013). Limitationen für den Einsatz des $\mathrm{CO}_{2}$-Lasers stellen anatomische Gegebenheiten dar. So können versteifte Halswirbel oder stark protrudiert stehende Oberkiefer-Frontzähne beim Exponieren des Tumors hinderlich sein (Rudert und Werner 2011).

Der Laserstrahl hat eine Wellenlänge von $10.600 \mathrm{~nm}$ und erreicht Temperaturen von mehreren hundert Grad, was zur Verdampfung des Gewebes führt (Dellian 2009; Rudert und Werner 2011). Seine Absorption durch das Gewebe ermöglicht es, ihn zur Zerlegung von ebendiesem zu nutzen (Strong und Jako 1972). Der Tumor wird mithilfe des Lasers in kleinen Portionen entfernt, was den bis dato geltenden Prinzipien der Onkologie, Tumoren im Ganzen zu entfernen, widerspricht (Rudert und Werner 2011).

In seltenen Fällen kann es nach einer laserchirurgischen Behandlung zu Komplikationen kommen, die insbesondere schwellungsbedingte Dyspnoe, Nachblutungen oder Emphyseme umfassen können (Steiner et al. 2013). 
Ziel der transoralen Lasermikrochirurgie ist der maximale Erhalt von gesundem Gewebe und damit einhergehend der Funktionserhalt des Larynx bei zudem bestmöglicher onkologischer Sicherheit (Steiner et al. 2013).

\subsubsection{Vorteile der TLM}

Die Lasermikrochirurgie hat sich zu einer akzeptierten Alternative zu den klassischen Therapiemöglichkeiten entwickelt, da sie eine geringere Morbidität und etliche weitere Vorteile aufweist (Ambrosch 2007). Die laserchirurgische Resektion eines Tumors ermöglicht dem Operateur ein blutungsarmes Operationsfeld, da durch die Hitze des Laserstrahls simultan Blut- und Lymphgefäße versiegelt werden (Rudert und Werner 2011). Ferner sind durch den geringen Blutverlust Bluttransfusionen nicht erforderlich (Steiner et al. 2013). Die stückweise Entfernung des Tumors garantiert außerdem eine individuelle Therapie, sodass Unter- oder Übertherapien bestmöglich vermieden werden (Rudert und Werner 2011). Darüber hinaus unterscheiden sich das Karbonisierungsverhalten von gesundem und entartetem Gewebe. Dies ermöglicht dem Operateur unter operationsmikroskopischer Kontrolle Tumorgewebe von gesundem Gewebe zu differenzieren. Das Schneiden mitten durch den Tumor hindurch ermöglicht die Bestimmung der Tiefeninfiltration, was einen wesentlichen Vorteil in der transoralen lasermikrochirurgischen Resektion darstellt (Steiner et al. 2013). Aufgrund des transoralen Zugangs wird gesundes Gewebe weniger traumatisiert, was eine schnellere Genesung zur Folge hat. Auch werden Tracheotomien seltener erforderlich und sind häufig nur temporär (Rudert und Werner 2011). Eine Wiederholung der Lasermikrochirurgie, beispielsweise im Fall eines Rezidivs oder bei einer nicht vollständig im Gesunden erfolgten Resektion, ist möglich. Insbesondere im Vergleich zur primären Radiotherapie, die meist über einen Zeitraum von sechs Wochen erfolgt, ist für den Patienten ein laserchirurgischer Eingriff mit einem kürzeren Krankenhausaufenthalt und einem schnelleren Wiedereinstieg in den Alltag verbunden. Des Weiteren lässt sich die transorale Lasermikrochirurgie in unterschiedliche Therapiekonzepte integrieren. Kombinationen mit einer Neck dissection oder rekonstruktionschirurgischen Verfahren sind ebenso möglich wie mit einer adjuvanten Radio(chemo)therapie (Steiner et al. 2013). 


\subsubsection{Nachteile der TLM}

Mit der TLM gehen auch Nachteile einher. Von Nachteil ist beispielsweise die verlängerte Wundheilungsdauer nach laserchirurgischer Behandlung. Im Durchschnitt dauert sie zehn Tage länger als nach einer klassischen Operation mit einem Skalpell, was den Einsatz beispielsweise in der Phonochirurgie limitiert (Rudert und Werner 2011). Ferner ist die Wirkung der mosaikartigen Resektion von Tumoren auf das Lymphsystem umstritten. Bei einem Tierexperiment konnten Sapundzhiev et al. (2005) zeigen, dass die portionsweise Entfernung eines Tumors mittels Laser im Vergleich zur klassischen Tumorentfernung im Ganzen zu einer erhöhten Lymphknotenmetastasierung führt (47,7\% Metastasen nach TLM, 24,6 \% nach klassischer Tumorentfernung). Werner et al. (1995) hingegen kommen $\mathrm{zu}$ dem Schluss, dass eine Tumorresektion unter Zuhilfenahme des $\mathrm{CO}_{2}$-Lasers eine Tumorzelleinschwemmung ins Lymphsystem nicht fördert. Wiegand et al. (2013) führten einen Tierversuch durch, in dem sie ein aurikuläres Plattenepithelkarzinom unterschiedlich therapierten. Es gab vier Therapiegruppen. In Gruppe A wurde der Tumor en bloc unter Zuhilfenahme eines Skalpells operiert. In Gruppe B erfolgte die Tumorentfernung im Ganzen mithilfe eines Lasers bei zwei Watt. In den Gruppen C beziehungsweise D wurde der Tumor portionsweise bei zwei beziehungsweise zwanzig Watt entfernt. In Gruppe A entwickelten 17,4 \% Metastasen in regionären Lymphknoten. In Gruppe B lag die Häufigkeit von Lymphknotenmetastasen bei 20,4\%. Bei der portionsweisen Tumorresektion in den Gruppen C und D entwickelten $26 \%$ beziehungsweise $20 \%$ Lymphknotenmetastasen. Wiegand et al. schlussfolgern, dass alle Behandlungsoptionen ein ähnlich hohes Risiko für postoperative Metastasen haben. Die Ausbreitung von Tumorzellen kann bei keiner der angewandten Operationsmethoden ausgeschlossen werden.

\subsubsection{Primäre Radio(chemo)therapie}

Die in der Strahlentherapie eingesetzte ionisierende Strahlung zur Behandlung von Tumoren führt zu Strangbrüchen der DNA der Zielzellen (van Kampen 2014). Bei Wiederholung der Bestrahlung und bei geringen Strahlendosen resultiert eine Proliferationshemmung und letztendlich der Zelltod des tumorösen Gewebes, während sich das gesunde Gewebe regenerieren kann (van Kampen 2014; Werner 2008).

Die normofraktionierte Einzeldosis liegt bei 1,8 bis 2,0 Gray (Gy) (van Kampen 2014). Die Gesamtdosis des Larynx liegt bei perkutaner konventioneller Radiatio bei circa 66 Gy 
(Pigorsch et al. 2009). Bei Überschreitungen werden Chondronekrosen beobachtet (Oehler 2014). Ferner berichten Hata et al. (2013) von akuten Toxizitäten nach Bestrahlungstherapien. Sie erwähnen in Bezug auf das hämatopoetische System Leukopenien, Anämien sowie Thrombozytopenien. Zudem können als Nebenwirkungen Erytheme und Mukositiden in bestrahlten Gebieten auftreten. Darüber hinaus können als Folge der Radiotherapie zervikale Lymphödeme, Xerostomie oder Osteoradionekrosen auftreten (Pigorsch et al. 2009).

Angewandt werden kann die Radiatio im Rahmen eines kurativen oder palliativen Behandlungskonzepts. Dabei können Primärtumore, Rezidive oder Metastasen behandelt werden (Pigorsch et al. 2009). Eine primäre Radiotherapie kann insbesondere bei Tumoren in frühen Stadien (T1-2, N0, M0) erfolgreich zum Einsatz kommen (Pigorsch et al. 2009; Dempke 2006). Bei lokal fortgeschrittenen Tumoren jedoch führt eine konventionell fraktionierte Radiotherapie zu vergleichsweise schlechten Ergebnissen. Eine Akzelerierung (Verkürzung der Gesamtbehandlungszeit) oder eine Hyperfraktionierung (Erhöhung der Gesamtdosis bei zeitgleicher Verringerung der Dosis pro Fraktion) kann zur Verbesserung der Tumorkontrolle fortgeschrittener Tumoren führen (Pigorsch et al. 2009).

Ein reduzierter Allgemeinzustand eines multimorbiden Patienten spricht gegen eine primäre Radiatio (Werner 2008; Pigorsch et al. 2009).

Um die lokale Tumorkontrollrate bei fortgeschrittenen Kopf-Hals-Malignomen zu erhöhen, werden auch kombinierte Radiochemotherapien herangezogen. Die Wirkungsweise der verschiedenen Zytostatika ist unterschiedlich. So wird die DNA-Reparatur von durch Strahlen geschädigten Zellen verhindert oder strahlenresistente Tumorzellen werden zerstört. Zudem können Zytostatika Tumorzellen für eine im Nachhinein folgende Radiatio sensibilisieren (Siefert et al. 2009). Als Zytostatika dienen 5-Fluoruracil, Cisplatin, Carboplatin, Mitomycin C sowie Hydroxidcarbamid (Werner 2008; Pignon et al. 2000).

\subsubsection{Laryngektomie}

Eine Laryngektomie beziehungsweise Kehlkopftotalentfernung wird dann in Betracht gezogen, wenn minimalinvasive Eingriffe missglückt sind beziehungsweise wenn durch diese keine Heilung erwartet wird (Schwab 1955). Einige Autoren verstehen unter der Laryngektomie lediglich eine rettungschirurgische Therapiemöglichkeit, die zur Rezidivbehandlung nach nicht erfolgreicher Primärtherapie dienen soll (Delank und Stoll 
2000; Young 2008). Mozet und Dietz (2010) geben als mögliche Indikationen für eine Laryngektomie folgende Kriterien an: „Infiltration des Ringknorpels oder der hinteren Kommissur, subglottisches Wachstum über den Ringknorpel hinaus, Tumordurchbruch im Bereich des gesamten Schildknorpels, transglottisches Wachstum in Richtung Supraglottis mit Infiltration des Zungenbeins beziehungsweise Anteilen des Zungengrunds, insbesondere bei beidseitigem Befall, beidseitiger Aryknorpelbefall, tiefe Infiltration des paraglottischen Raumes mit kompletter Stimmlippenfixierung (relative Indikation), nach fehlgeschlagener primärer Radio- beziehungsweise Radiochemotherapie und vorbestehender Indikation zur Laryngektomie (Salvage), nach überzogener Indikation der Teilresektion und bei Chondroradionekrose des Larynx (auch ohne Tumornachweis).“

Durch die Laryngektomie werden der obere und untere Luftweg voneinander getrennt, folglich sind die oberen Atemwege nicht mehr an der Atmung beteiligt. Ferner wird auch die Verbindung zwischen Luft- und Speiseweg aufgehoben. Die Atmung erfolgt über ein dauerhaftes Tracheostoma (Schwab 1955; Mozet und Dietz 2010). Durch die Trennung zwischen Luft- und Speiseweg ist die Gefahr der Aspiration nicht gegeben, auch wird der Schluckvorgang nicht erschwert (Delank und Stoll 2000). Erheblich geschwächt werden durch den fehlenden Luftstrom im oberen Atemweg jedoch Riech- und Geschmackssinn (Mozet und Dietz 2010). Aus der Kehlkopftotalentfernung resultiert zudem auch der Stimmverlust. Es werden Stimmersatzverfahren erforderlich. Etwa $60 \%$ aller Laryngektomierten erlernen die Ösophagusersatzstimme, welche durch Schwingungen der Ösophagusschleimhaut zustande kommt. Auch besteht die Möglichkeit, das Sprechen durch eine Stimmprothese, die über ein Einwegventil Atemluft in den Pharynx pressen lässt oder durch elektronische Sprechhilfen (Elektrolarynx) wiederzuerlangen (Delank und Stoll 2000). Die häufigste Komplikation nach einer Laryngektomie ist das Auftreten einer Fistel als Folge einer Wundheilungsstörung (Delank und Stoll 2000).

Erstmalig führte der Arzt Theodor Billroth 1873 eine totale Laryngektomie an einem Larynxkarzinom-Patienten in Wien durch, nachdem einen Monat zuvor die teilweise Entfernung des Larynx missglückt war. Sieben Monate später verstarb der erste Laryngektomierte vermutlich durch eine Metastase (Genden et al. 2007; Karamanou et al. 2017). Heute findet die Laryngektomie zu Gunsten organerhaltender Verfahren seltener Anwendung, ist aber stets in vielen der oben genannten Situationen eine notwendige und onkologisch sichere Therapieoption. In der Bundesrepublik leben zurzeit schätzungsweise 20.000 kehlkopflose Menschen (Mozet und Dietz 2010). 


\subsubsection{Adjuvante Radio(chemo)therapie}

Eine adjuvante Radio(chemo)therapie kann nach erfolgter Primärbehandlung durchgeführt werden.

Der Einsatz einer adjuvanten Radiatio hängt vom individuellen Rezidivrisiko, der Lokalisation und Größe des Primärtumors, den Resektionsrändern sowie dem Befall lokoregionärer Lymphknoten ab. In der Regel werden die Patienten mit Gesamtdosen von 50 bis 60 Gy bestrahlt. Auf sie kann verzichtet werden, wenn sehr frühe Tumorstadien (T12, N0) vorliegen und die Resektion im Gesunden (R0) erreicht wurde. Tumoren der T3- oder T4-Kategorie sollten neben einer Operation auch mit einer adjuvanten Radiatio oder Radiochemotherapie behandelt werden (Pigorsch et al. 2009; DKG 2019). Bei fortgeschrittenen Tumoren ohne Metastasierung besteht auch die Option einer primären Bestrahlung mit gegebenenfalls anschließender Salvage-Operation (DKG 2019).

Eine adjuvante Radiochemotherapie beinhaltet neben der lokalen auch die Komponente der systemischen Therapie. Sie wird bei fortgeschrittenen Primärtumoren, bei Lymphknotenbefall oder zur Verbesserung der lokoregionären Kontrolle in Erwägung gezogen. Eine Kombination aus Radiatio und Chemotherapie erhöht den Therapieeffekt, verursacht jedoch auch akute Nebenwirkungen (Schmoll et al. 1997).

Verglichen mit einer alleinigen Radiotherapie werden mit einer simultanen Radiochemotherapie postoperativ signifikant höhere Raten lokoregionärer Tumorfreiheit bei fortgeschrittenen Kopf-Hals-Tumoren erzielt (Bernier et al. 2004). Auch Cooper et al. (2004) kommen in ihrer Studie zu dem Schluss, dass eine Kombinationstherapie zu einer besseren lokoregionären Tumorkontrolle bei Kopf-Hals-Karzinomen führt als eine alleinige Radiotherapie.

\subsubsection{Neck dissection}

Unter einer Neck dissection versteht man die Ausräumung des fibroadipösen und die Lymphknoten und Lymphgefäße beinhaltenden Gewebes im Halsbereich, um bei Malignomen im Kopf-Hals-Bereich Lymphknotenmetastasen zu behandeln (Cornelius et al. 2009). Sie kann bei klinischem Nachweis von Lymphknotenmetastasen in kurativer Absicht erfolgen. Sollte nach klinischer Erfahrung mit einem Lymphknotenbefall zu rechnen sein, sich dieser aber klinisch noch nicht zeigen, erfolgt eine elektive beziehungsweise prophylaktische Neck dissection. 
Lymphknotenmetastasen treten häufig bei Plattenepithelkarzinomen der Mund-, Rachenund Kehlkopfschleimhaut auf. Der Hals wird dabei topographisch in sechs Level unterteilt, in die ein Primärtumor metastasieren kann. Primärtumoren des Larynx metastasieren häufig in die Level II, III sowie IV (Cornelius et al. 2009). Im Durchschnitt geht mit supraglottisch lokalisierten Malignomen am häufigsten ein Lymphknotenbefall einher (30\% bis $50 \%$ ), gefolgt von subglottischen (20\% bis $35 \%$ ) und glottischen (0\% bis $10 \%$ ) Malignomen (Schwab und zum Winkel 1975).

\subsubsection{Tumornachsorge}

Die Tumornachsorge beginnt unmittelbar nach Beendigung der Primärtherapie und enthält neben der Anamnese auch klinische Untersuchungen. Im Anamnesegespräch wird auf eventuelle Beschwerden des Patienten näher eingegangen. Die klinische Untersuchung beinhaltet eine lokale inspektorisch und heute endoskopisch gestützte Untersuchung der Schleimhäute sowie die Palpation der Halslymphknoten. Regelmäßig sollte zudem eine Sonographie der Halsweichteile durchgeführt werden, ergänzend nach festgelegtem Schema beispielsweise im Abstand von ein und zwei Jahren nach abgeschlossener Tumortherapie oder bei Rezidivverdacht auch eine Computertomographie oder Magnet-ResonanzTomographie (Papachristofilou et al. 2009).

Mehr als zwei Drittel aller Lokalrezidive im Kopf-Hals-Bereich werden innerhalb der ersten zwei Jahre nach Abschluss der Primärbehandlung im Rahmen der Tumornachsorge diagnostiziert (Haas 2000). Ziele der Tumornachsorge sind es, neben der Kontrolle des onkologischen Therapieerfolgs, Nebenwirkungen der Therapie zu ermitteln sowie gegebenenfalls auch die Wiederherstellung der Phonations- und Schluckfunktion aktiv zu begleiten. Darüber hinaus soll dem Patienten die Angst vor Rezidiven genommen werden. Er soll sensibilisiert werden, bei selbst festgestellten Veränderungen unverzüglich einen Arzt aufzusuchen. Die Tumornachsorge beinhaltet auch den Aspekt der Vorsorge, da in ihrem Rahmen Zweitmalignome ausgeschlossen werden können (Haas 2000).

Das empfohlene Untersuchungsintervall der Nachsorge liegt bei drei Monaten in den ersten drei Jahren nach abgeschlossener Primärtherapie, im Anschluss erfolgt die Nachsorge halbjährlich (Papachristofilou et al. 2009). 


\subsection{Ziel der Studie}

Im Rahmen der Studie werden onkologische und funktionelle Ergebnisse verschiedener Therapiestrategien zur Behandlung der seltenen Entität von Plattenepithelkarzinomen der Subglottis evaluiert. Dazu werden die Ergebnisse der zwischen 1992 und 2013 an der Universitätsmedizin Göttingen behandelten Patienten erhoben und mit Kollektiven aus anderen Studien verglichen.

Zielparameter der retrospektiven Untersuchung sind für die onkologischen Ergebnisse die lokale Tumorkontrolle, das Gesamtüberleben, das krankheitsspezifische Überleben und das rezidivfreie Überleben. Zur Untersuchung des funktionellen Erfolgs werden die Organerhaltungsrate sowie Komplikationen näher betrachtet.

Es existieren keine prospektiven Studien, welche die modernen Therapiemöglichkeiten bei subglottischen Plattenepithelkarzinomen untersuchen (Levy et al. 2014), was die Notwendigkeit retrospektiver Untersuchungen weiterhin unterstreicht. 


\section{Patienten und Methode}

\subsection{Ethik}

Die vorliegende retrospektive Studie wurde am 10.01.2017 unter der Antragsnummer DOK_177_2016 von der Ethik-Kommission der Universitätsmedizin Göttingen genehmigt.

\subsection{Datenerhebung}

Die Datenerhebung erfolgte retrospektiv mithilfe von Suchanfragen in elektronischen Datenbanken und der Analyse von Patientenakten. Weitere Nachforschungen bei den Patienten selbst wurden nicht durchgeführt.

Dokumentiert wurden neben dem Geschlecht und Alter der Patienten auch deren Krankengeschichte und das Vorhandensein von Zweittumoren. Berücksichtigung fanden in Bezug auf das subglottische Malignom das Diagnosedatum, die exakte subglottische Ausdehnung, das histologische Grading und die TNM-Kategorie. Darüber hinaus erfolgte eine genaue Dokumentation der angewandten Therapie, einschließlich eventuell durchgeführter Neck dissection oder Radio(chemo)therapie und daraus resultierenden Komplikationen. Das Anlegen von Nasogastral-, perkutanen endoskopischen GastrostomieSonden (PEG) und Tracheostomas wurde in der Datenerhebung ebenfalls aufgezeigt. Zudem wurden auftretende Rezidive und ihre Therapie beschrieben.

\subsection{Patientenkollektiv}

Zur Identifikation potenzieller Fälle mit einem primär subglottischen Karzinom erfolgte eine Abfrage über den entsprechenden ICD-10 (International Statistical Classification of Diseases and Related Health Problems) Code C32.2 (bösartige Neoplasie der Subglottis) durch das MedizinControlling. Darüber hinaus wurden Fälle über die abteilungsinterne Tumordatenbank sowie mittels Stichwortabfrage in der Datenbank des Instituts für Pathologie identifiziert. Insgesamt wurden auf diese Weise 126 Patientenfälle ermittelt, die anhand der Patientenakte auf Erfüllung der Ein- und Ausschlusskriterien hin untersucht wurden. Von den 126 identifizierten Fällen standen 123 Patientenakten zur Auswertung zur Verfügung. Drei 
Patientenakten wurden nach Ablauf der gesetzlich vorgeschriebenen Archivierungsfrist vernichtet.

\subsubsection{Einschlusskriterien}

Es wurden ausschließlich die Fälle in die Studie aufgenommen, die ein primär subglottisches Plattenepithelkarzinom aufwiesen.

Die Tumorentität musste histologisch einwandfrei gesichert sein. Darüber hinaus musste die subglottische Lokalisation des Primärtumors anhand des Operationsberichts zweifelsfrei nachvollziehbar sein. Der Operateur definierte bei Ausdehnung über die Subglottis hinaus die Primärlokalisation des Tumors.

\subsubsection{Ausschlusskriterien}

Von den 123 identifizierten Fällen mit verfügbarer Patientenakte ergab die detaillierte Sichtung den Ausschluss von 106 (86 \%) Fällen. Der häufigste Ausschlussgrund ( $\mathrm{n}=87$ ) war das Vorliegen eines nicht primär subglottischen Tumors. Meist handelte es sich um primär glottische Tumoren, die die Subglottis infiltrierten. Weitere Gründe für einen Ausschluss waren in elf Fällen ein vorangegangener Primärtumor im Kopf-Hals-Bereich sowie eine andere Histologie als das Plattenepithelkarzinom $(n=9)$. Ferner wurden Patienten ausgeschlossen, die sich zur Rezidivtherapie in die Universitätsmedizin Göttingen begaben, deren Primärtherapie jedoch andernorts erfolgte. Hiervon wurde in neun Fällen auswärts lasermikrochirurgisch behandelt, in vier Fällen erfolgte alio loco eine primäre Radio(chemo)therapie, in vier weiteren Fällen war die Vorbehandlung nicht bekannt. In zwei Fällen führten simultane Zweittumoren und in zwei weiteren Fällen bereits vorliegende Fernmetastasen zum Ausschluss aus dem Kollektiv. In einem Fall war ein palliatives Therapiekonzept der Ausschlussgrund. Von den 123 identifizierten Fällen mit verfügbarer Patientenakte erfüllten 17 (13\%) alle Ein- und keine Ausschlusskriterien und wurden für die weitere Auswertung in der Studie berücksichtigt. 


\subsection{Staging und Nachbeobachtungszeitraum}

Das Tumor-Staging wurde unter Berücksichtigung der siebten Edition der TNMKlassifizierung der Union for International Cancer Control durchgeführt (Edge und Compton 2010; Sobin und Compton 2010). Während des evaluierten Behandlungszeitraums änderte sich das Staging subglottischer Tumore nicht erheblich. Mit der sechsten Auflage fand eine Untergruppierung der T4-Kategorie in T4a und T4b statt. Weil im Patientenkollektiv kein Tumor der T4b-Kategorie (Tumor infiltriert den Prävertebralraum, mediastinale Strukturen oder umschließt die Arteria carotis interna) diagnostiziert wurde, hat die Änderung keine Auswirkung auf die vorliegende Studie.

Nach Abschluss der Therapie erfolgte eine systematische Tumornachsorge, in dessen Zuge die Patienten in den ersten drei Jahren alle drei Monate vorstellig werden sollten, im Anschluss für weitere zwei Jahre alle sechs Monate. Nach fünf Jahren wurde ein Patient als von der Erkrankung geheilt angesehen. Die Nachsorge erfolgte in den meisten Fällen darüber hinaus im jährlichen Rhythmus weiter.

\subsection{Statistik}

Zur Berechnung der Überlebensstatistiken wurde die Kaplan-Meier-Methode mit einem Konfidenzintervall von $95 \%$ herangezogen (Kaplan und Meier 1958). Dabei wurde der Beobachtungszeitraum als Zeit zwischen dem Operationstag des Primärtumors und dem letzten Kontakt zum Patienten beziehungsweise des Todestags definiert. Folgende Kenngrößen wurden mithilfe der Kaplan-Meier-Methode berechnet: Lokale Tumorkontrolle (Local control rate, LCR), Fünf-Jahres-Gesamtüberleben (Overall survival, OS), Fünf-Jahres krankheitsspezifisches Überleben (Disease specific survival, DSS) und rezidivfreies Überleben (Recurrence free survival, RFS).

Zur Ermittlung der lokalen Tumorkontrollrate wurden ausschließlich Lokalrezidive als Ereignisse gewertet, zensiert wurde der Tod jedweder Art oder lebend ohne Lokalrezidiv.

Beim Fünf-Jahres-Gesamtüberleben zählten Todesursachen jedweder Art als Ereignis, zensiert wurden alle lebenden Patienten.

Als Event beim Fünf-Jahres krankheitsspezifischen Überleben zählte ausschließlich der Tod durch ein subglottisches Karzinom. Zensiert wurden lebende, interkurrent Verstorbene oder der zweittumorbedingte Tod. 
$\mathrm{Zu}$ den Ereignissen beim rezidivfreien Überleben gehörten Lokalrezidive, Lymphknotenmetastasen, Fernmetastasen sowie der Tod durch ein subglottisches Malignom, während interkurrent Verstorbene, zweittumorbedingter Tod oder lebende Patienten ohne Rezidiv zensiert wurden.

Als Residualtumoren wurden solche Malignome gewertet, welche bis zu drei Monate nach Primärtherapie auftraten. Nach mehr als drei Monaten handelte es sich um ein Rezidiv.

Trat eine regionäre Metastase nach zunächst klinisch oder histologisch negativem Lymphknotenbefall (cN0/pN0) auf, wurde sie als Spätmetastase bezeichnet. Um eine rezidivierend auftretende Metastase handelte es sich nach chirurgischer Initialtherapie und histologisch abgesichertem Lymphknotenbefall (pN1).

Der finale Krankheitsstatus beim letzten Patientenkontakt wurde definiert als: lebend ohne Tumor (auf den Primärtumor bezogen), lebend mit Tumor, interkurrent verstorben, TNMbedingt verstorben (auf den Primärtumor bezogen) oder zweittumorbedingt verstorben.

Die statistische Analyse erfolgte mit dem Programm Dell Statistica Version 12 (Dell, Round Rock, Texas). 


\section{Ergebnisse}

\subsection{Alters- und Geschlechtsverteilung}

Das durchschnittliche Alter lag bei 58,7 Jahren, der Median betrug 61 Jahre. Der jüngste Patient war 32, der älteste 77 Jahre alt. Alle Patienten waren männlich, weibliche Patienten waren in der Kohorte nicht vertreten.

\subsection{Nachbeobachtung}

Die mittlere Nachsorgeperiode der Kohorte lag bei 69,5 Monaten, im Durchschnitt betrug sie 60,9 Monate. Die Zeitspanne der Nachbeobachtung erstreckte sich von 1,1 bis 212,8 Monate.

\subsection{Verteilung der TNM-Kategorien}

Zum Zeitpunkt der Diagnose hatten jeweils vier Patienten einen pT2- beziehungsweise pT3Tumor. Beim Großteil des Kollektivs war der Tumor bereits weit fortgeschritten und entsprach der pT4a-Kategorie $(\mathrm{n}=9)$. In keinem Fall wurde ein T1-Tumor diagnostiziert, somit war kein Tumor ausschließlich auf die Subglottis beschränkt.

In sechs Fällen lag zum Diagnosezeitpunkt eine regionäre Metastasierung vor. Die Tabellen 5 und 6 geben einen Überblick über die Verteilung der T- beziehungsweise N-Kategorien, Tabelle 7 zeigt die Häufigkeitsverteilung der T- und N-Kategorien zueinander. 
Tabelle 5: Primärtumor - Verteilung der T-Kategorien

\begin{tabular}{|l|l|c|}
\hline pT-Kategorie & $\begin{array}{l}\text { Anzahl der } \\
\text { Patienten }\end{array}$ & {$[\%]$} \\
\hline 1 & 0 & 0 \\
2 & 4 & 23,5 \\
3 & 4 & 23,5 \\
$4 \mathrm{a}$ & 9 & 53,0 \\
\hline Gesamt & 17 & 100 \\
\hline
\end{tabular}

Tabelle 6: Halslymphknotenmetastasierung - Verteilung der N-Kategorien

\begin{tabular}{|l|l|c|}
\hline pN-Kategorie & $\begin{array}{l}\text { Anzahl der } \\
\text { Patienten }\end{array}$ & \\
\hline 0 & 11 & 64,7 \\
1 & 3 & 17,6 \\
$2 \mathrm{a}$ & 0 & 0 \\
$2 \mathrm{~b}$ & 2 & 11,8 \\
$2 \mathrm{c}$ & 1 & 5,9 \\
3 & 0 & 0 \\
\hline Gesamt & 17 & 100 \\
\hline
\end{tabular}

Tabelle 7: Primärtumor - Kombination der T- und N-Kategorien

\begin{tabular}{|l|l|l|l|l|l|l|l|}
\hline pT-Kategorie & c/pN0 & pN1 & pN2a & pN2b & pN2c & pN3 & Gesamt \\
\hline pT1 & 0 & 0 & 0 & 0 & 0 & 0 & 0 \\
pT2 & 4 & 0 & 0 & 0 & 0 & 0 & 4 \\
pT3 & 2 & 1 & 0 & 1 & 0 & 0 & 4 \\
pT4a & 5 & 2 & 0 & 1 & 1 & 0 & 9 \\
\hline Gesamt & 11 & 3 & 0 & 2 & 1 & 0 & 17 \\
\hline
\end{tabular}




\subsection{Verteilung der UICC-Stadien}

Da in keinem Fall ein Carcinoma in situ oder ein Tumor im T1-Stadium diagnostiziert wurde, sind die UICC-Stadien 0 und I nicht vertreten. Vier Fälle aus dem Kollektiv sind aufgrund des vorliegenden T2-Tumors ohne zervikale Metastasierung dem UICC-Stadium II zugeordnet. Drei Fälle entsprechen dem Stadium III, bei einem T3-Tumor in zwei Fällen und einem T3-Tumor mit singulärer ipsilateraler Lymphknotenmetastase (N1) in einem Fall. Der Hauptanteil $(\mathrm{n}=10)$ verteilt sich auf Stadium IVa, da entweder ein Tumor der Kategorie T4a oder ein Lymphknotenbefall von N2 (oder eine Kombination aus beidem) vorliegt. Tabelle 8 zeigt die Verteilung der UICC-Stadien.

Tabelle 8: Verteilung der UICC-Stadien

\begin{tabular}{|l|c|}
\hline $\begin{array}{l}\text { UICC- } \\
\text { Stadium }\end{array}$ & $\begin{array}{l}\text { Anzahl } \\
\text { der } \\
\text { Patienten }\end{array}$ \\
\hline 0 & 0 \\
I & 0 \\
II & 4 \\
III & 3 \\
IV & 10 \\
\hline Gesamt & 17 \\
\hline
\end{tabular}




\subsection{Therapie des Primärtumors}

Die Patienten der vorliegenden Auswertung wurden zwischen 1992 und 2013 mit einem primär subglottischen Plattenepithelkarzinom in der Abteilung für Hals-NasenOhrenheilkunde der Universitätsmedizin Göttingen kurativ therapiert. Für die 17 eingeschlossenen Fälle wurden unterschiedliche Therapiestrategien herangezogen. In den meisten Fällen $(\mathrm{n}=6)$ erfolgte ausschließlich die transorale lasermikrochirurgische Tumorresektion, gefolgt von TLM und einer Neck dissection $(\mathrm{n}=5)$. Die TLM in Kombination mit einer Neck dissection und adjuvanter Radio(chemo)therapie erfolgte in zwei Fällen. In drei Fällen bestand das Therapiekonzept aus einer totalen Laryngektomie in Kombination mit einer Neck dissection und adjuvanten Radio(chemo)therapie. Mit nur einem Fall am seltensten erfolgte eine primäre Radiochemotherapie $(\mathrm{n}=1)$. Zusammenfassend erfolgte die Therapie von subglottischen Larynxkarzinomen mit 13 Fällen (76\%) überwiegend mittels minimalinvasiver transoraler Lasermikrochirurgie. Die folgende Abbildung 2 zeigt einen Überblick zur Verteilung der Therapiestrategien.

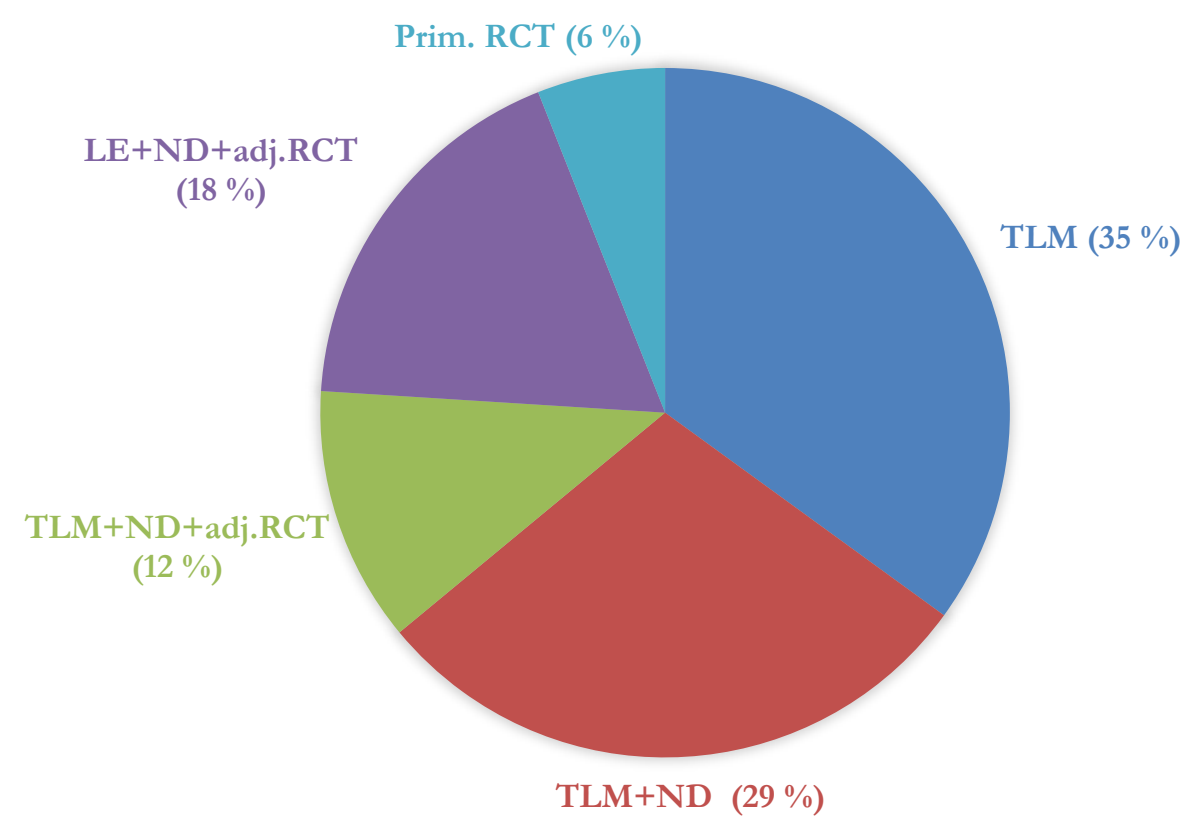

Abbildung 2: Therapiestrategien des Primärtumors (TLM: Transorale Lasermikrochirurgie, ND: Neck dissection, adj. RCT: adjuvante Radio(chemo)therapie, LE: Laryngektomie, Prim. RCT: Primäre Radiochemotherapie)

Betrachtet man die Therapie des Primärtumors bezogen auf die T-Kategorie, zeigt sich, dass bei lokal umschriebenen Tumoren (pT2) ausschließlich eine transorale lasermikrochirurgische Tumorresektion erfolgte. Bei einem pT3-Tumor wurde die TLM 
meist durch eine Neck dissection und in einem Fall auch zusätzlich durch eine adjuvante Radiochemotherapie ergänzt. Bei weiter fortgeschritten Tumoren (p'T4a) erfolgte die Behandlung in einigen Fällen ebenfalls primär mittels TLM, zudem wurden Patienten auch durch eine primäre Radiochemotherapie oder totale Laryngektomie behandelt.

Die laserchirurgisch behandelten pT4a-Fälle wurden ebendieser Kategorie zugeordnet, weil der Tumor bereits die Trachea infiltrierte $(\mathrm{n}=2)$ oder sich im Weichgewebe des Halses $(n=2)$ oder in Trachea und dem Weichgewebe des Halses $(n=1)$ ausgebreitet hatte. Primär laryngektomierte Fälle wurden der pT4a-Kategorie zugewiesen, weil der Tumor bereits die Trachea infiltrierte $(\mathrm{n}=1)$, das Weichgewebe des Halses infiltriert war $(\mathrm{n}=1)$ oder neben dem Halsweichgewebe auch der Schildknorpel tumorös verändert war $(n=1)$. Ein cT4a-Fall mit Befall der Halsweichgewebe und des Ösophagus wurde mit primärer Radiochemotherapie behandelt.

Die Tabellen 9 und 10 zeigen die Behandlungsstrategien in Abhängigkeit von der Größe des Primärtumors (T-Kategorie) und dem UICC-Stadium. Die Abbildungen 3 bis 5 veranschaulichen den prozentualen Anteil der drei primären Therapiestrategien an den Tumorgrößen.

Tabelle 9: Therapie des Primärtumors in Abhängigkeit von der T-Kategorie (TLM: Transorale Lasermikrochirurgie, ND: Neck dissection, adj. RCT: adjuvante Radiochemotherapie, Prim. RCT: Primäre Radiochemotherapie, LE: Laryngektomie)

\begin{tabular}{|l|l|l|l|l|l|}
\hline Therapie & pT1 & pT2 & pT3 & pT4a & Gesamt \\
\hline TLM & 0 & 4 & 1 & 1 & 6 \\
TLM+ND & 0 & 0 & 2 & 3 & 5 \\
TLM+adj. RCT & 0 & 0 & 0 & 0 & 0 \\
TLM+ND+adj.RCT & 0 & 0 & 1 & 1 & 2 \\
Prim. RCT & 0 & 0 & 0 & 1 & 1 \\
LE & 0 & 0 & 0 & 0 & 0 \\
LE+ ND+RCT & 0 & 0 & 0 & 3 & 3 \\
\hline Gesamt & 0 & 4 & 4 & 9 & 17 \\
\hline
\end{tabular}


Tabelle 10: Therapie des Primärtumors in Abhängigkeit vom UICC-Stadium (TLM: Transorale Lasermikrochirurgie, ND: Neck dissection, RCT: Radiochemotherapie, Prim. RCT: Primäre Radiochemotherapie, LE: Laryngektomie)

\begin{tabular}{|l|c|c|c|c|c|}
\hline Therapie & UICC I & UICC II & UICC III & UICC IV & Gesamt \\
\hline TLM & 0 & 4 & 1 & 1 & 6 \\
TLM+ND & 0 & 0 & 1 & 4 & 5 \\
TLM+RCT & 0 & 0 & 0 & 0 & 0 \\
TLM+ND+RCT & 0 & 0 & 1 & 1 & 2 \\
Prim. RCT & 0 & 0 & 0 & 1 & 1 \\
LE+ND+RCT & 0 & 0 & 0 & 3 & 3 \\
\hline Gesamt & 0 & 4 & 3 & 10 & 17 \\
\hline
\end{tabular}

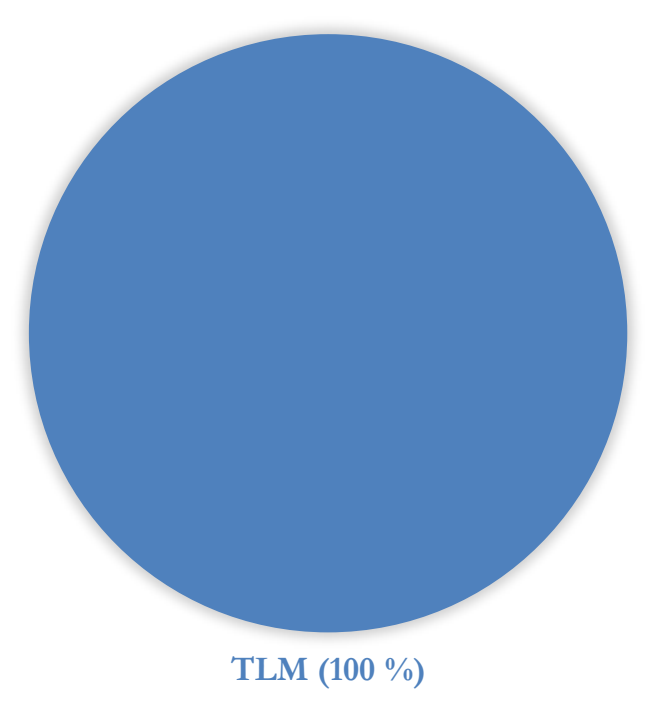

Abbildung 3: Therapiestrategie bei T2-Tumoren (TLM: Transorale Lasermikrochirurgie) 


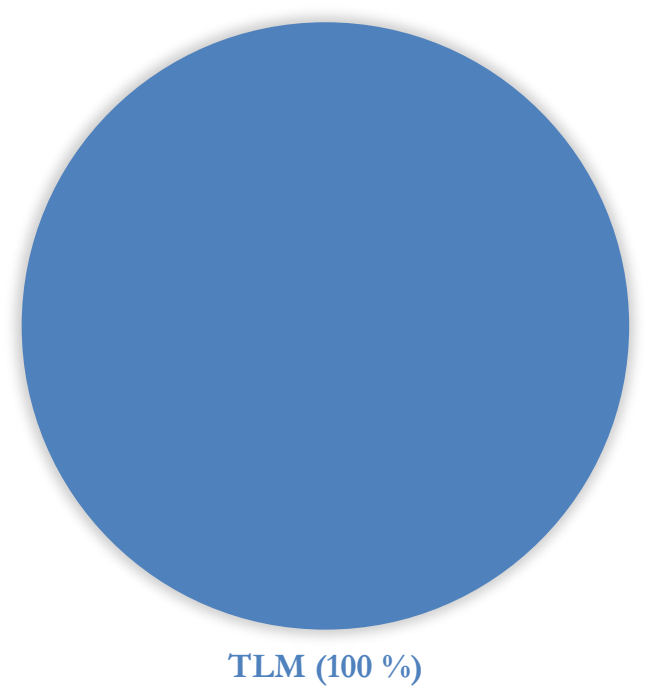

Abbildung 4: Therapiestrategie bei T3-Tumoren (TLM: Transorale Lasermikrochirurgie)

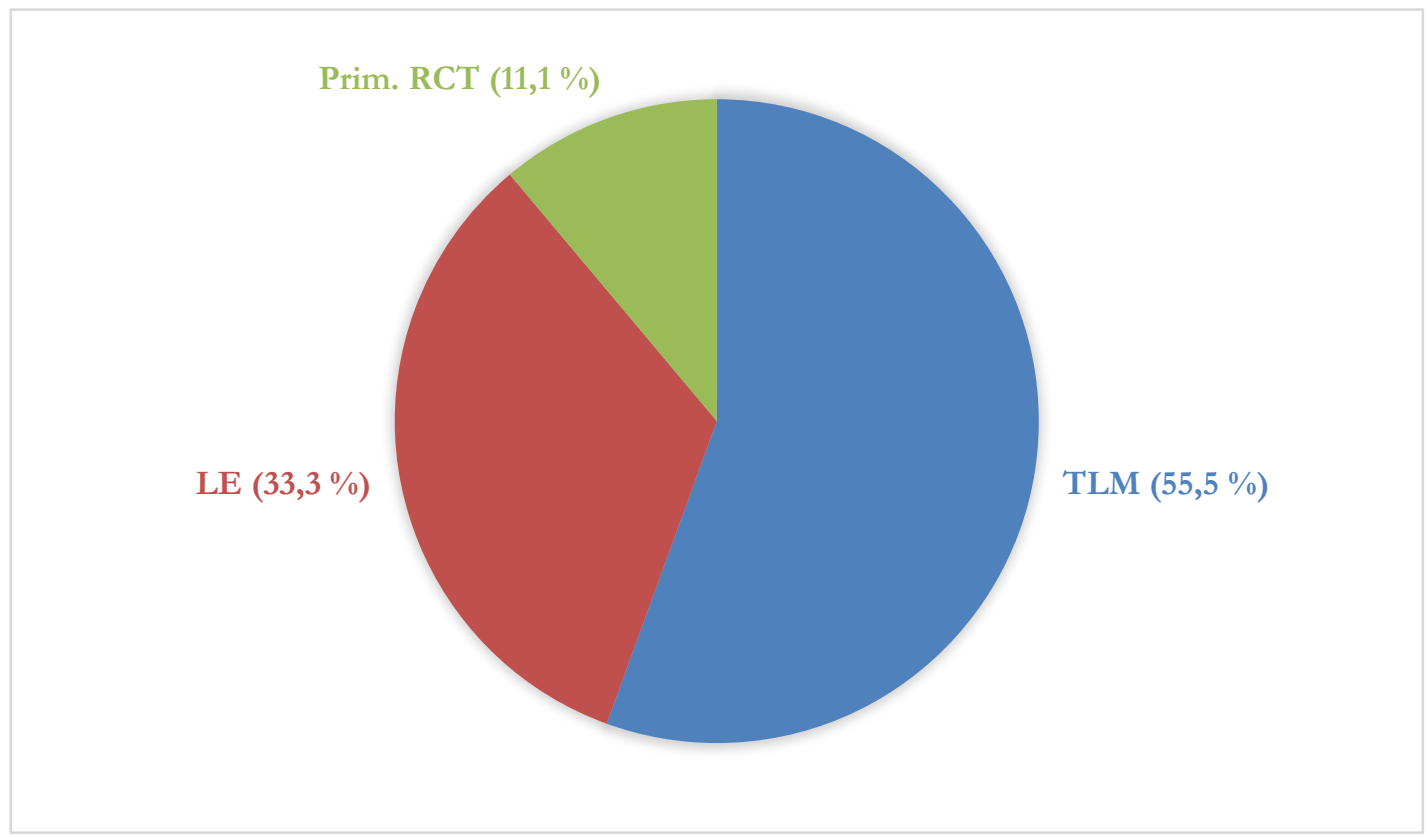

Abbildung 5: Therapiestrategie bei T4-Tumoren (TLM: Transorale Lasermikrochirurgie, LE: Laryngektomie, Prim. RCT: Primäre Radiochemotherapie) 


\subsubsection{Adjuvante Radio(chemo)therapie}

Bei fünf Patienten erfolgte im Rahmen des kurativen Therapiekonzepts eine adjuvante Radio(chemo)therapie. Davon erhielten drei eine alleinige Radiotherapie mit Gesamtdosen zwischen 60 und 64 Gy (inklusive eines Boosts für die Primärtumorregion). Bestrahlt wurden neben der Primärtumorregion auch der regionäre Lymphabfluss inklusive Supraclavicularregion.

Bei zwei weiteren Patienten erfolgte zusätzlich zur adjuvanten Radiotherapie eine begleitende Chemotherapie mit dem Zytostatikum Cisplatin.

\subsubsection{Neck dissection}

Von den laserchirurgisch therapierten Patienten $(\mathrm{n}=13)$ wurde in sieben Fällen zusätzlich eine selektive Neck dissection (53,8 \%) durchgeführt, in 15,4\% der Fälle ( $\mathrm{n}=2$ ) erfolgte sie unilateral, in 38,4 \% $(\mathrm{n}=5)$ bilateral. Von den zwölf präparierten Halsseiten beinhalteten neun die Level II, III und IV. Zudem wurden Level I und/oder Level VI in zwei beziehungsweise vier Halsseiten chirurgisch ausgeräumt. Ausschließlich Level II und III wurden auf einer Halsseite, nur Level VI auf beiden Halsseiten präpariert. Insgesamt erfolgte in sechs von zwölf Halsseiten (50\%) eine Neck dissection in Level VI (drei Patienten mit bilateraler Ausräumung von Level VI, alle drei waren an einem Tumor der Kategorie pT4a erkrankt, zwei davon mit Infiltration der Halsweichgewebe). Eine radikale oder modifiziert radikale Neck dissection wurde nicht durchgeführt. Von den laryngektomierten Patienten ( $\mathrm{n}=3$ ) erhielten alle eine bilaterale Neck dissection. Werden alle chirurgisch behandelten Fälle betrachtet, ergab die histologische Bewertung einen positiven Lymphknotenbefall in fünf von zehn durchgeführten Neck dissections (50\%). Ein Patient (10\%) war bilateral von regionären Metastasen betroffen.

Bei sechs Patienten mit einem cN0-Status erfolgte keine elektive Neck dissection. Diese zeigten im Verlauf auch keine Lymphknotenmetastasierung beziehungsweise Spätmetastasen. Insgesamt lag der Anteil der bei Erstdiagnose und nach chirurgischer Therapie metastasierten Fälle bei 31,2 \% (fünf der chirurgisch therapierten Patienten).

Eine Salvage-Neck dissection nach vorausgegangener primärer Radiochemotherapie wurde im vorliegenden Kollektiv nicht beobachtet. 


\subsection{Zweittumoren und relevante Nebendiagnosen}

Aus dem vorliegenden Gesamtkollektiv ( $\mathrm{n}=17)$ erkrankten fünf Patienten (29\%) im Laufe der Nachbeobachtung an einem Zweittumor. Die Zeit von Behandlung des Primärtumors bis zur Diagnosestellung des Zweittumors reichte von 18 bis 153 Monate (ein bis elf Jahre, im Durchschnitt 87 Monate). Die Zweittumoren waren in zwei Fällen (12\%) in der Lunge lokalisiert. Zwei Patienten erkrankten an einem Zweittumor der Kopf-Hals-Region, davon einer erneut in der Subglottis (nach elf Jahren Tumorfreiheit) und einer im Hypopharynx (sechs Jahre nach Primärtherapie des subglottischen Tumors). Bei einem Patienten (6\%) trat ein Colonkarzinom auf.

Aus der Kohorte waren drei Patienten bereits vor der Diagnose des subglottischen Karzinoms an einem bösartigen Tumor erkrankt. Hierbei handelte es sich um ein Bronchialkarzinom (zwei Jahre vor Diagnose des subglottischen Karzinoms), ein Prostatakarzinom (sieben Jahre vor Diagnose des subglottischen Karzinoms) und ein malignes Melanom (drei Monate vor Diagnose des subglottischen Karzinoms).

\subsection{Onkologische Ergebnisse}

\subsubsection{Onkologische Ergebnisse zum Zeitpunkt der letzten Tumornachsorge}

Zum letzten Nachuntersuchungszeitpunkt lebte der Großteil der untersuchten Kohorte tumorfrei $(\mathrm{n}=12 ; 71 \%)$. Drei Patienten waren interkurrent, also an einer anderen Ursache als das Tumorleiden verstorben. Jeweils ein Patient war TNM-bedingt verstorben oder lebte mit einem Rezidiv des subglottischen Karzinoms. Im Folgenden werden die onkologischen Resultate zum Zeitpunkt der letzten Tumornachsorge tabellarisch dargestellt (Tabelle 11). 
Tabelle 11: Onkologische Ergebnisse zum Zeitpunkt der letzten Tumornachsorge

\begin{tabular}{|l|l|l|}
\hline Status & $\begin{array}{l}\text { Anzahl } \\
\text { der } \\
\text { Patienten }\end{array}$ & [\%] \\
\hline Lebt ohne Tumor & 12 & 70,6 \\
\hline Lebt mit Tumor (auf Primärtumor bezogen) & 1 & 5,9 \\
\hline Interkurrent verstorben & 3 & 17,6 \\
\hline Verstorben aufgrund der Primärerkrankung & 1 & 5,9 \\
\hline Zweittumorbedingt verstorben & 0 & 0 \\
\hline Gesamt & 17 & 100 \\
\hline
\end{tabular}

\subsubsection{Therapieversager}

In der 17 Patienten zählenden Kohorte erlitten sechs (35\%) ein Rezidiv, davon fünf ein Lokalrezidiv und einer ein lokoregionäres Rezidiv. Im Mittel vergingen bis zum Auftreten des ersten Rezidivs 20,6 Monate (Zeitspanne: 4,8 bis 50,9 Monate). Die T-Kategorie des ersten Rezidivs war in fünf Fällen rT4a, ein Patient hatte einen rT2-Tumor. Zur Therapie dieses ersten Rezidivs erfolgte in drei Fällen eine Laryngektomie, zwei Patienten wurden erneut lasermikrochirurgisch behandelt. Bei einem Patienten mit kutanen Metastasen erfolgte eine palliative Behandlung.

Zwei der fünf kurativ behandelten Patienten mit einem Rezidiv entwickelten ein zweites Rezidiv. In einem Fall handelte es sich um einen rT4a-Tumor, in dem anderen Fall lag eine hepatische Metastase (M1) vor. Therapiert wurde der erste Fall mit einer endolaryngealen Teilresektion des Larynx mit Einbeziehung der Trachea mithilfe der transoralen Lasermikrochirurgie in Kombination mit einer adjuvanten Radiotherapie. Im zweiten Fall wurde der Patient palliativ behandelt. Für die von Rezidiven betroffenen sechs Patienten war der Status beim letzten Kontakt wie folgt: Drei Patienten lebten ohne Tumor (auf Primärtumor bezogen), ein Patient lebte mit Tumor (auf Primärtumor bezogen), ein Patient starb TNM-bedingt und ein weiterer verstarb interkurrent.

Die Tabellen 12 und 13 zeigen die Häufigkeiten und Art des ersten beziehungsweise zweiten Rezidivs. 
Tabelle 12: Häufigkeit und Art des ersten Rezidivs

\begin{tabular}{|l|l|l|}
\hline Art des Rezidivs & $\begin{array}{l}\text { Anzahl } \\
\text { der } \\
\text { Patienten }\end{array}$ & {$[\%]$} \\
\hline Lokalrezidiv & 5 & 83 \\
\hline Lokoregionäres Rezidiv & 1 & 17 \\
\hline Spätmetastase & 0 & 0 \\
\hline Rezidivmetastase & 0 & 0 \\
\hline Lokalrezidiv und Fernmetastase & 0 & 0 \\
\hline Fernmetastase & 0 & 0 \\
\hline Gesamt & 6 & 100 \\
\hline
\end{tabular}

Tabelle 13: Häufigkeit und Art des zweiten Rezidivs

\begin{tabular}{|l|l|l|}
\hline Art des Rezidivs & $\begin{array}{l}\text { Anzahl } \\
\text { der } \\
\text { Patienten }\end{array}$ & {$[\%]$} \\
\hline Lokalrezidiv & 1 & 33,3 \\
\hline Lokoregionäres Rezidiv & 0 & 0 \\
\hline Spätmetastase & 0 & 0 \\
\hline Rezidivmetastase & 0 & 0 \\
\hline Lokalrezidiv und Fernmetastase & 1 & 33,3 \\
\hline Fernmetastase & 1 & 33,3 \\
\hline Gesamt & 3 & 100 \\
\hline
\end{tabular}




\section{8 Überlebenszeitanalysen}

\subsubsection{Lokale Tumorkontrolle}

Die lokale Kontrollrate zum Zeitpunkt von fünf Jahren lag für das Gesamtkollektiv der 17 Patienten mit einem subglottischen Plattenepithelkarzinom bei 54,4\%. Folglich wurde bei $46 \%$ der Patienten innerhalb der ersten fünf Jahre ein Lokalrezidiv diagnostiziert.

Für die 13 primär lasermikrochirurgisch behandelten Patienten lag die Fünf-Jahres lokale Kontrollrate bei $46,4 \%$.

Abbildung 6 zeigt die Kaplan-Meier-Schätzung der lokalen Kontrollrate für das Gesamtkollektiv der primär lasermikrochirurgisch $(\mathrm{n}=13)$, primär radiochemotherapeutisch $(\mathrm{n}=1)$ sowie mittels totaler Laryngektomie $(\mathrm{n}=3)$ behandelten Patienten. In Abbildung 7 ist die die Kaplan-Meier-Schätzung der lokalen Kontrollrate ausschließlich für die 13 primär lasermikrochirurgisch behandelten Fälle aufgezeigt.

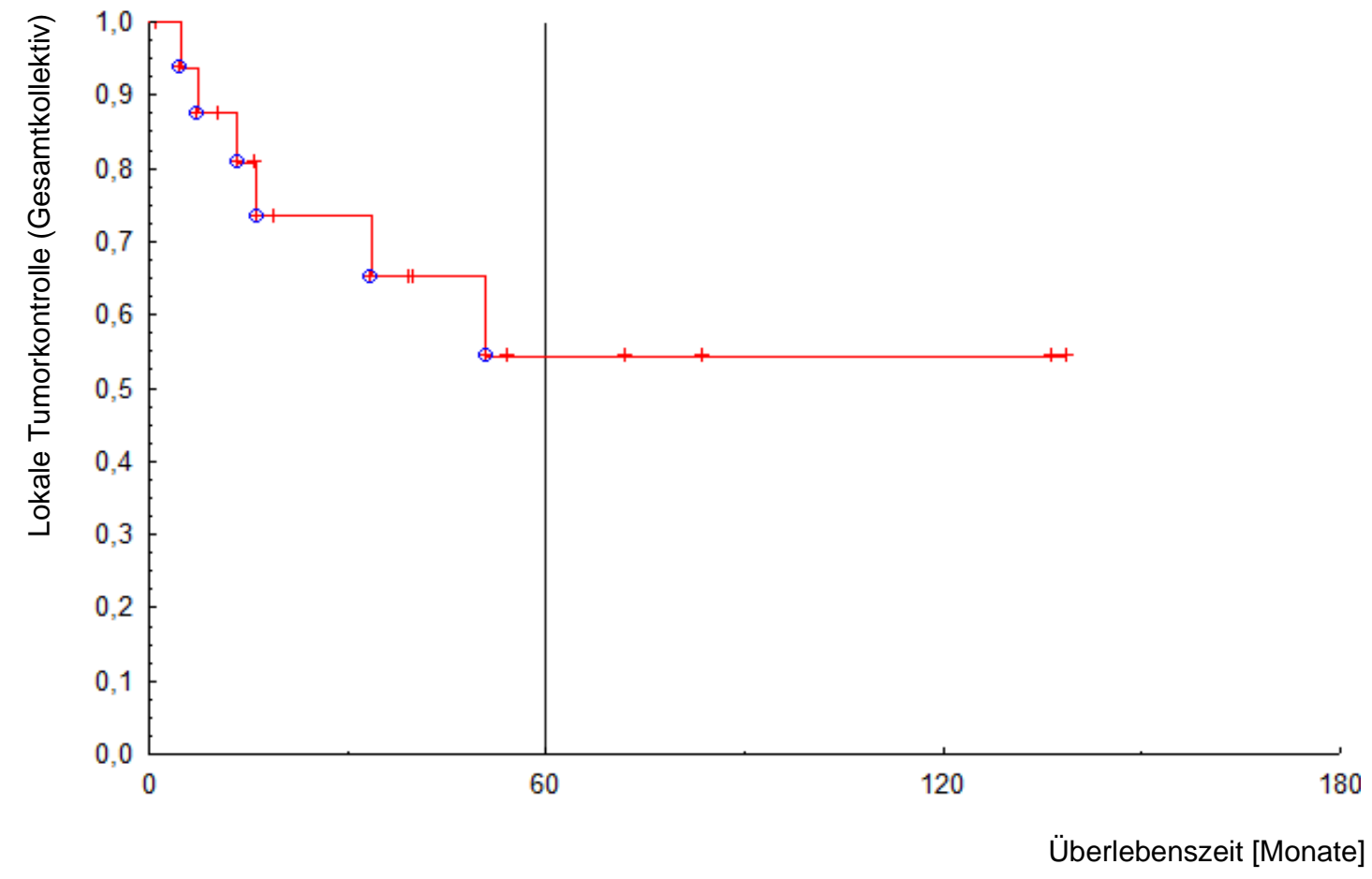

Abbildung 6: Kaplan-Meier-Schätzung für die lokale Kontrollrate des Gesamtkollektivs (alle Therapiestrategien) 


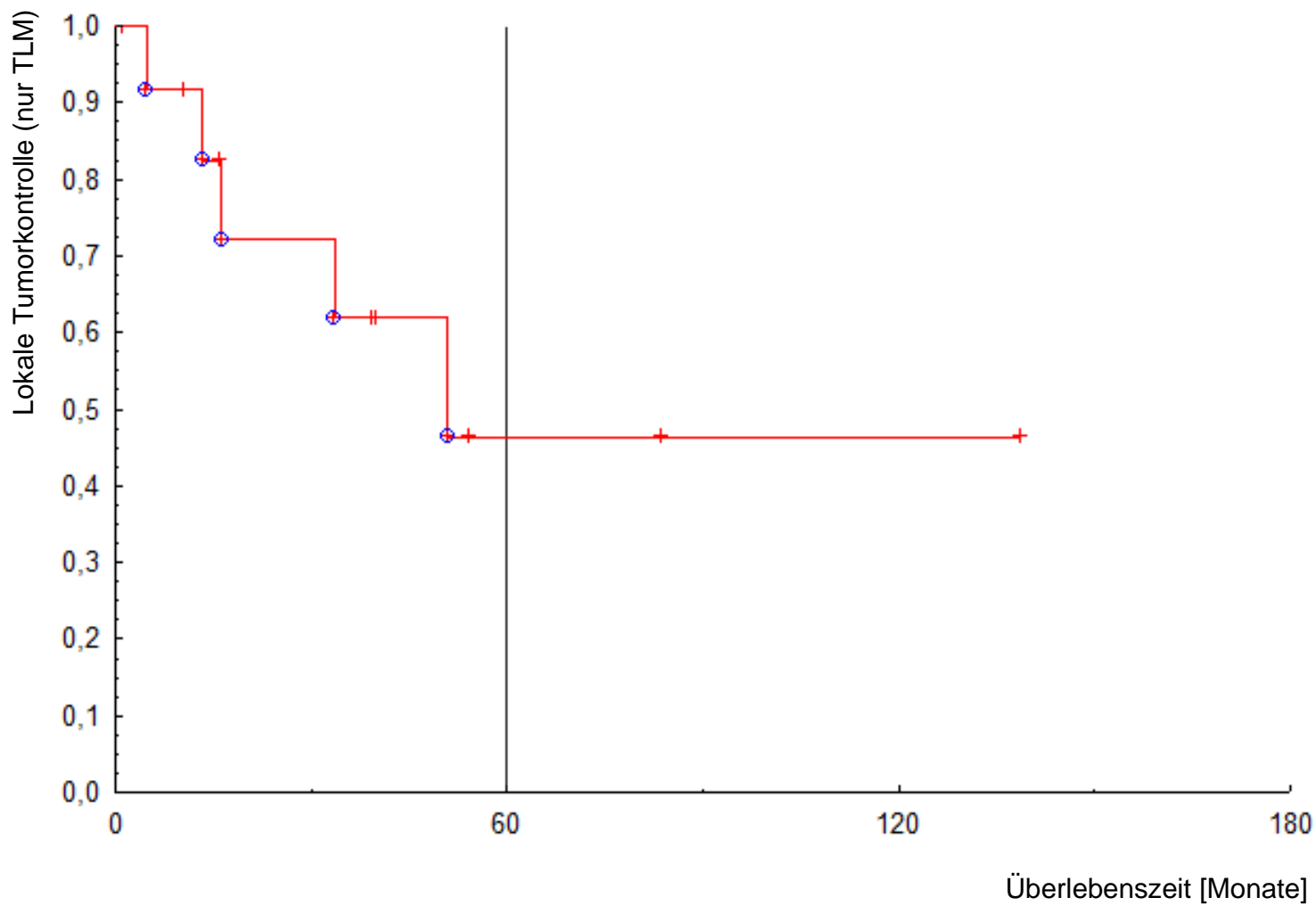

Abbildung 7: Kaplan-Meier-Schätzung für die lokale Kontrollrate der primär lasermikrochirurgisch behandelten Patienten 


\subsubsection{Fünf-Jahres-Gesamtüberleben}

Das Fünf-Jahres-Gesamtüberleben des Gesamtkollektivs lag bei 84,4 \%. Das Fünf-JahresGesamtüberleben für die 13 primär lasermikrochirurgisch behandelten Patienten lag bei $78,8 \%$.

Abbildung 8 zeigt die Kaplan-Meier-Schätzung des Gesamtüberlebens für das Gesamtkollektiv der primär lasermikrochirurgisch $(n=13)$, primär radiochemotherapeutisch $(\mathrm{n}=1)$ sowie mittels totaler Laryngektomie $(\mathrm{n}=3)$ behandelten Patienten. In Abbildung 9 ist die die Kaplan-Meier-Schätzung des Gesamtüberlebens ausschließlich für die 13 primär lasermikrochirurgisch behandelten Fälle aufgezeigt.

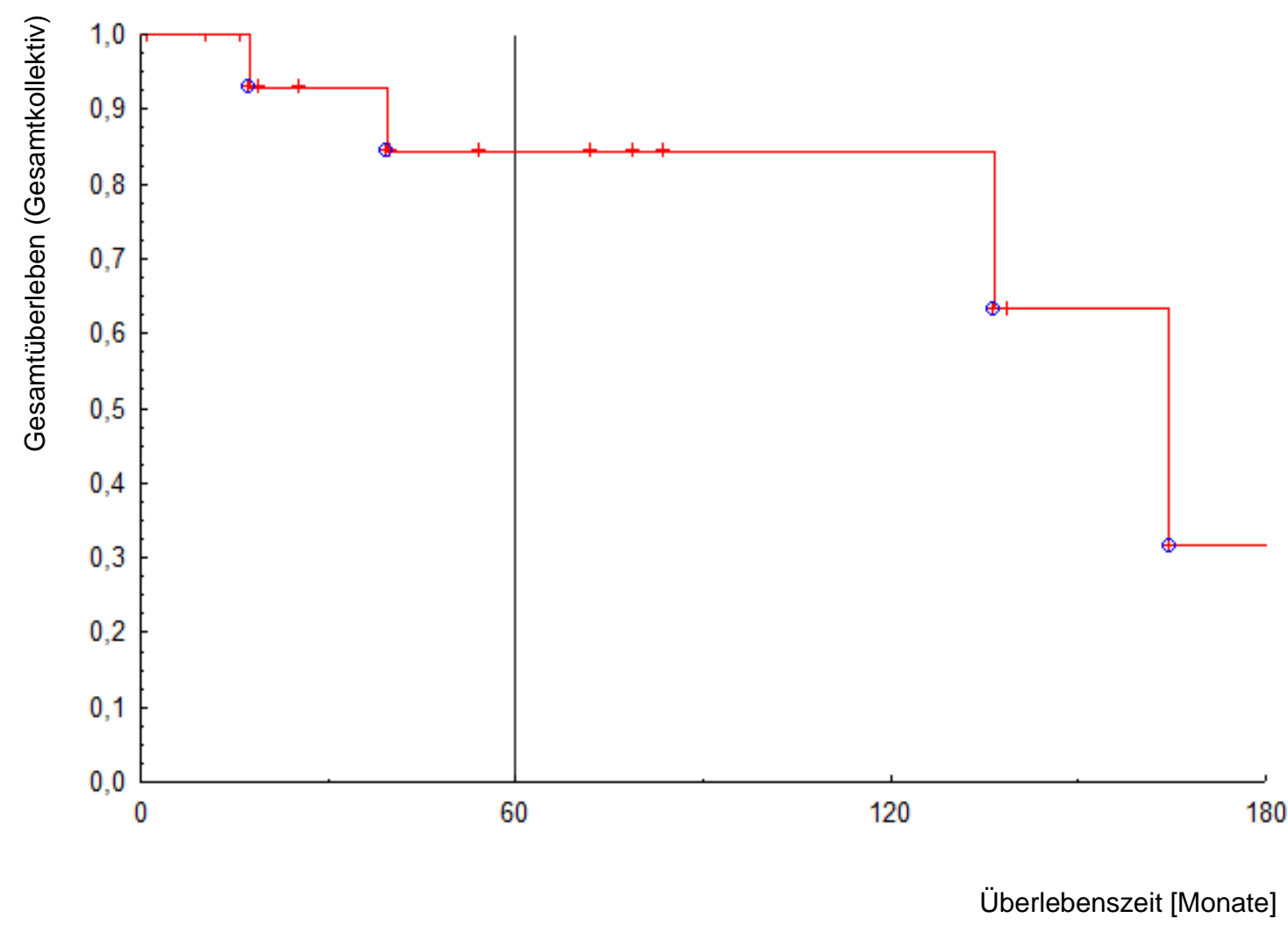

Abbildung 8: Kaplan-Meier-Schätzung für das Gesamtüberleben des Gesamtkollektivs (alle Therapiestrategien) 


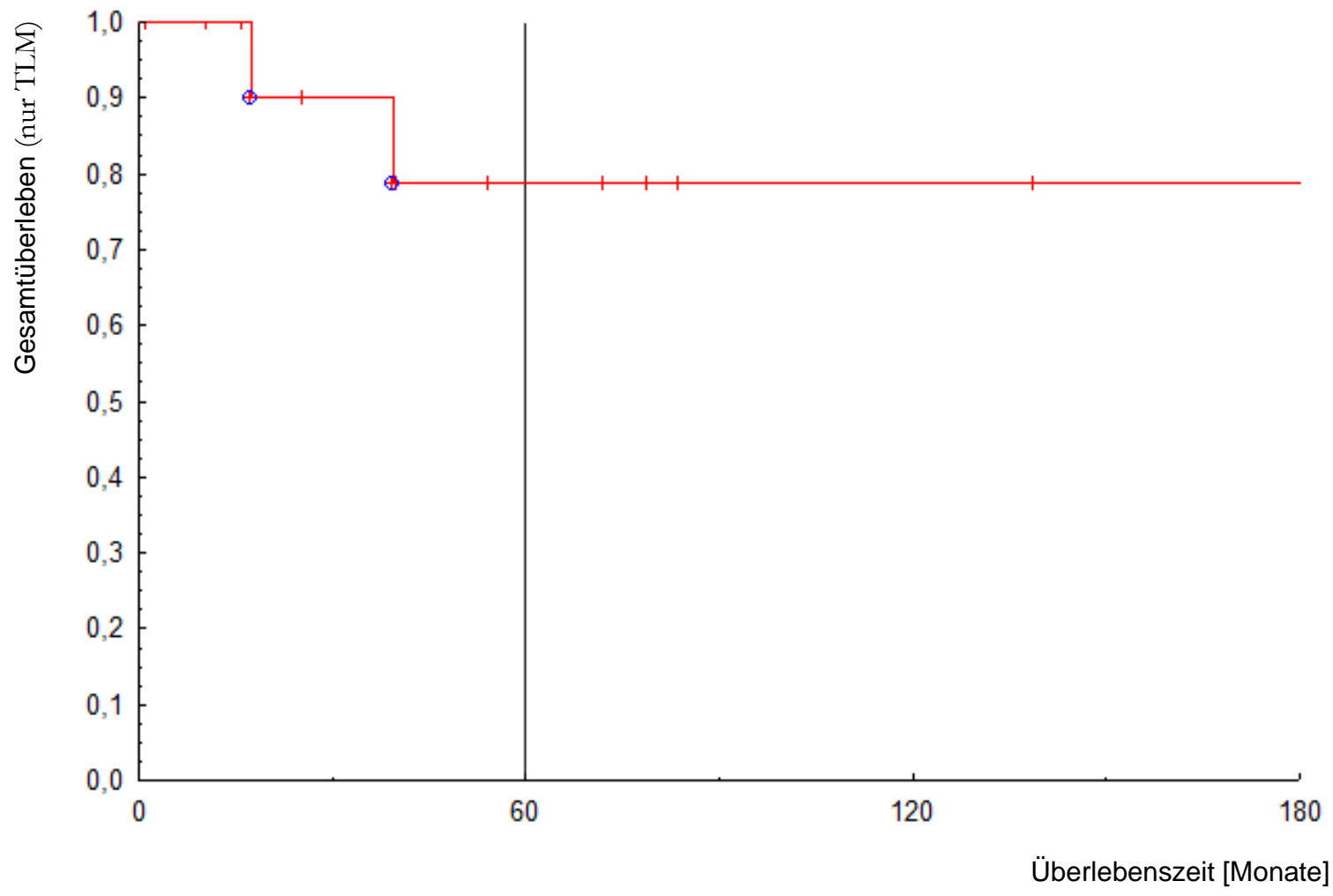

Abbildung 9: Kaplan-Meier-Schätzung für das Gesamtüberleben der primär lasermikrochirurgisch behandelten Patienten 


\subsubsection{Fünf-Jahres krankheitsspezifisches Überleben}

Das krankheitsspezifische Überleben lag in der vorliegenden Studie nach fünf Jahren bei $92,9 \%$, folglich sind circa $7 \%$ innerhalb der ersten fünf Jahre aufgrund eines subglottischen Tumors verstorben.

Das Fünf-Jahres krankheitsspezifische Überleben der primär lasermikrochirurgisch behandelten Patienten lag bei 90,0\%.

Abbildung 10 zeigt die Kaplan-Meier-Schätzung des krankheitsspezifischen Überlebens für das Gesamtkollektiv der primär lasermikrochirurgisch $(\mathrm{n}=13)$, primär radiochemotherapeutisch $(\mathrm{n}=1)$ sowie mittels totaler Laryngektomie $(\mathrm{n}=3)$ behandelten Patienten. In Abbildung 11 ist die Kaplan-Meier-Schätzung des krankheitsspezifischen Überlebens ausschließlich für die 13 primär lasermikrochirurgisch behandelten Fälle aufgezeigt.

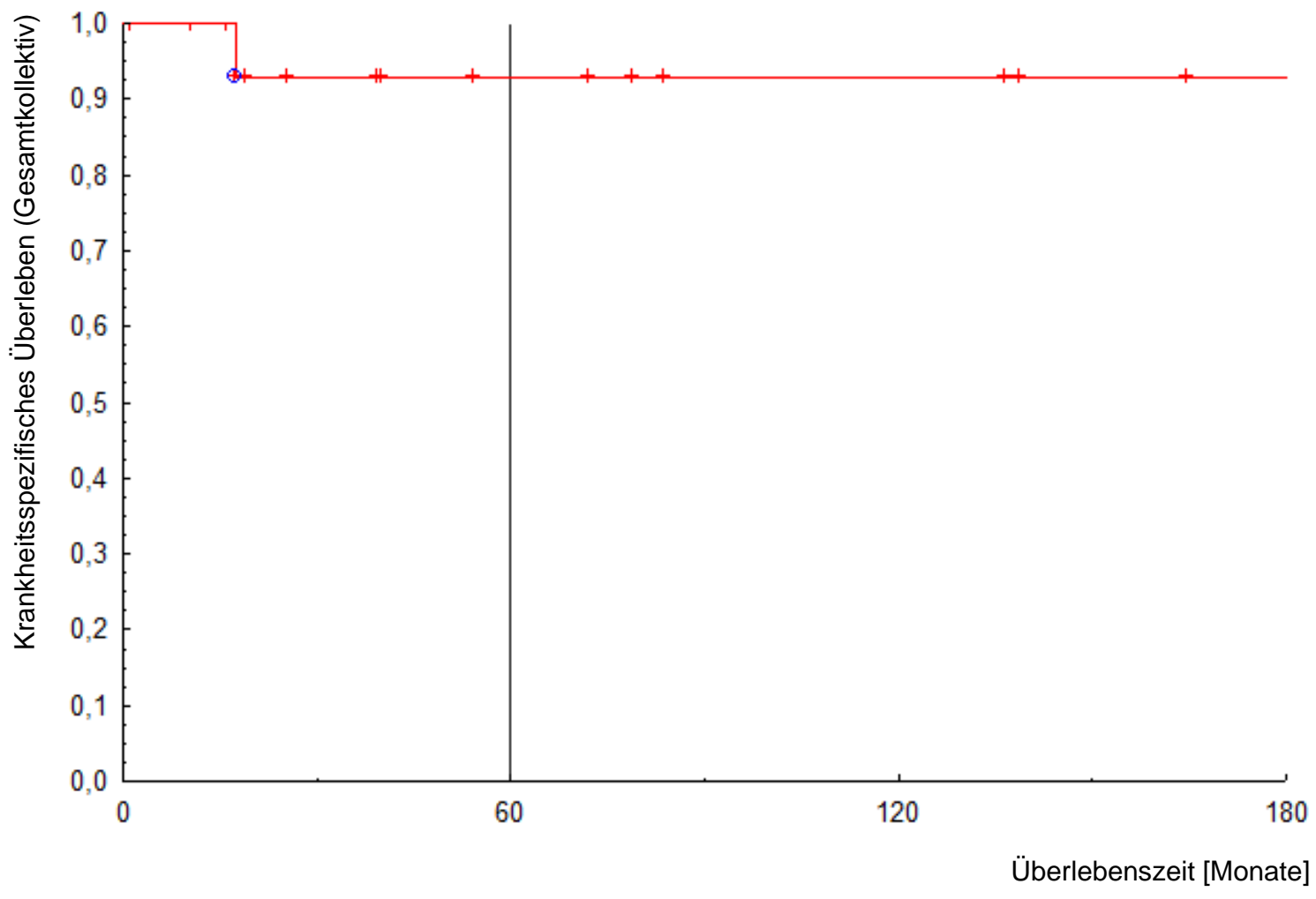

Abbildung 10: Kaplan-Meier-Schätzung für das krankheitsspezifische Überleben des Gesamtkollektivs (alle Therapiestrategien) 


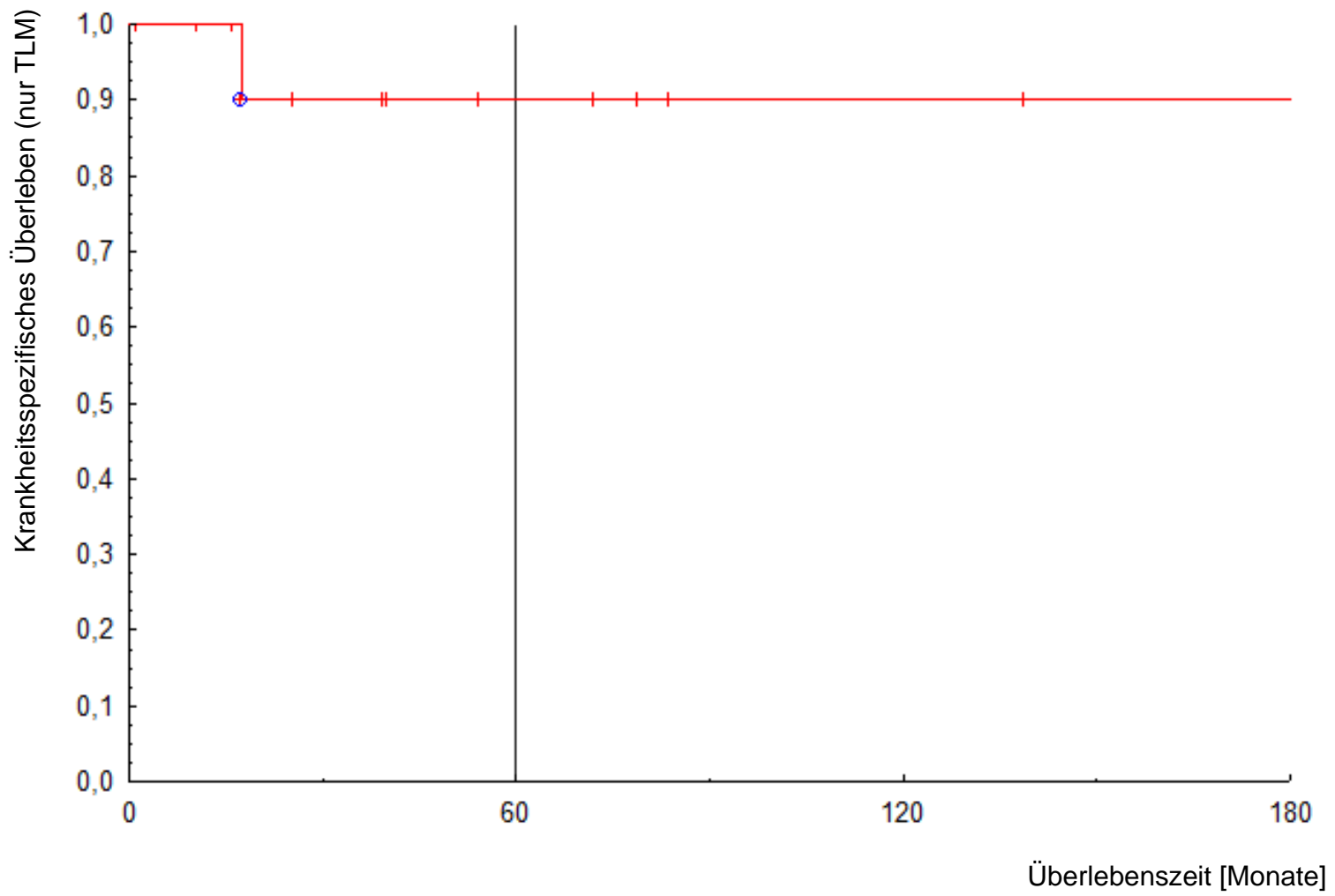

Abbildung 11: Kaplan-Meier-Schätzung für das krankheitsspezifische Überleben der primär lasermikrochirurgisch behandelten Patienten 


\subsubsection{Rezidivfreies Überleben}

Zum Zeitpunkt von fünf Jahren lag das rezidivfreie Überleben für das Gesamtkollektiv der 17 Patienten mit einem subglottischen Plattenepithelkarzinom bei 54,4\%. Folglich wurde bei $46 \%$ der Patienten innerhalb der ersten fünf Jahre ein lokoregionäres Rezidiv, eine Fernmetastase oder der Tod infolge des Primärtumors diagnostiziert.

Für die 13 primär lasermikrochirurgisch behandelten Patienten lag das Fünf-Jahres rezidivfreie Überleben bei 46,4\%.

Abbildung 12 zeigt die Kaplan-Meier-Schätzung des rezidivfreien Überlebens für das Gesamtkollektiv der primär lasermikrochirurgisch $(n=13)$, primär radiochemotherapeutisch $(\mathrm{n}=1)$ sowie mittels totaler Laryngektomie $(\mathrm{n}=3)$ behandelten Patienten.

In Abbildung 13 ist die Kaplan-Meier-Schätzung des rezidivfreien Überlebens ausschließlich für die 13 primär lasermikrochirurgisch behandelten Fälle aufgezeigt.

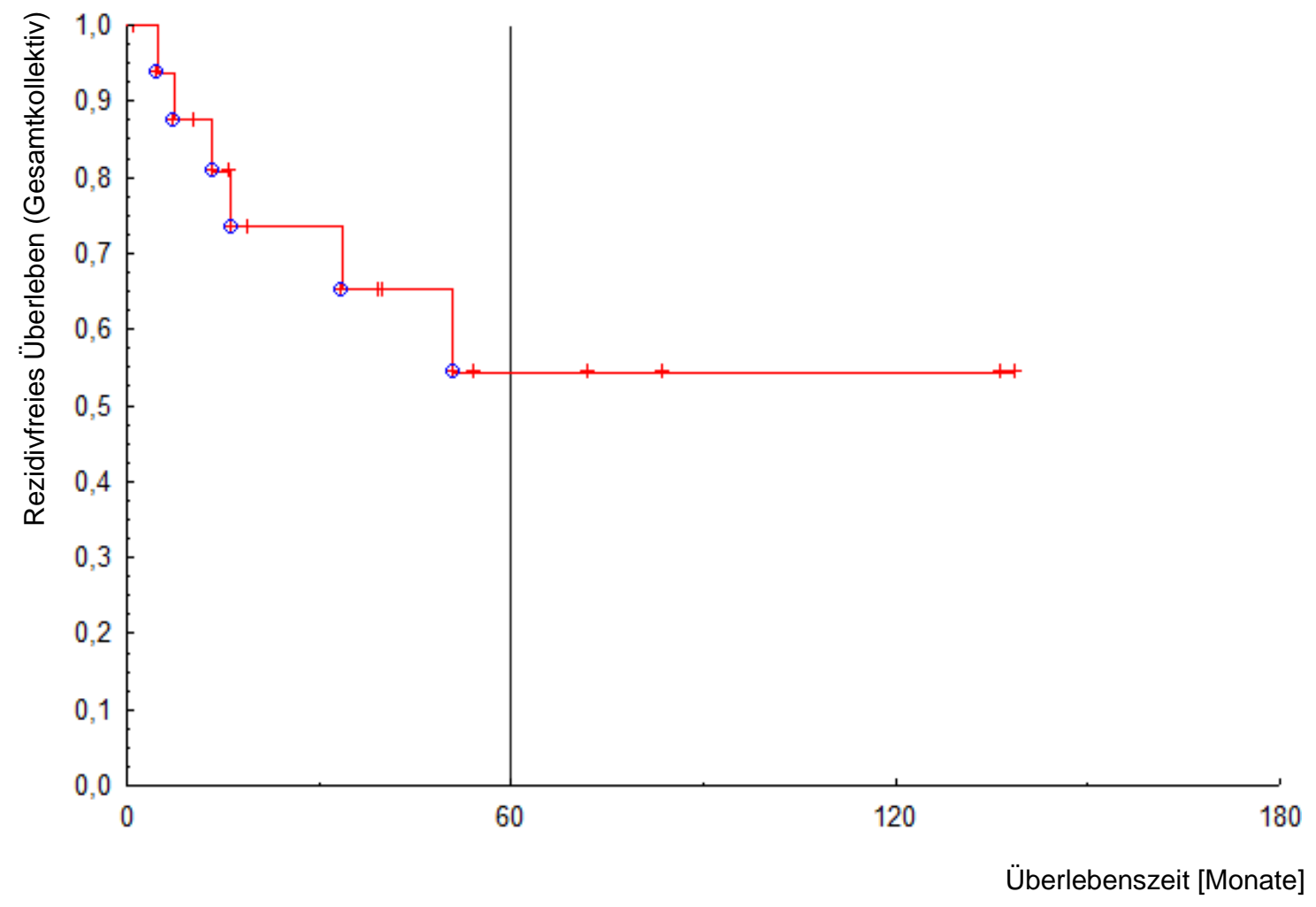

Abbildung 12: Kaplan-Meier-Schätzung für das rezidivfreie Überleben des Gesamtkollektivs (alle Therapiestrategien) 


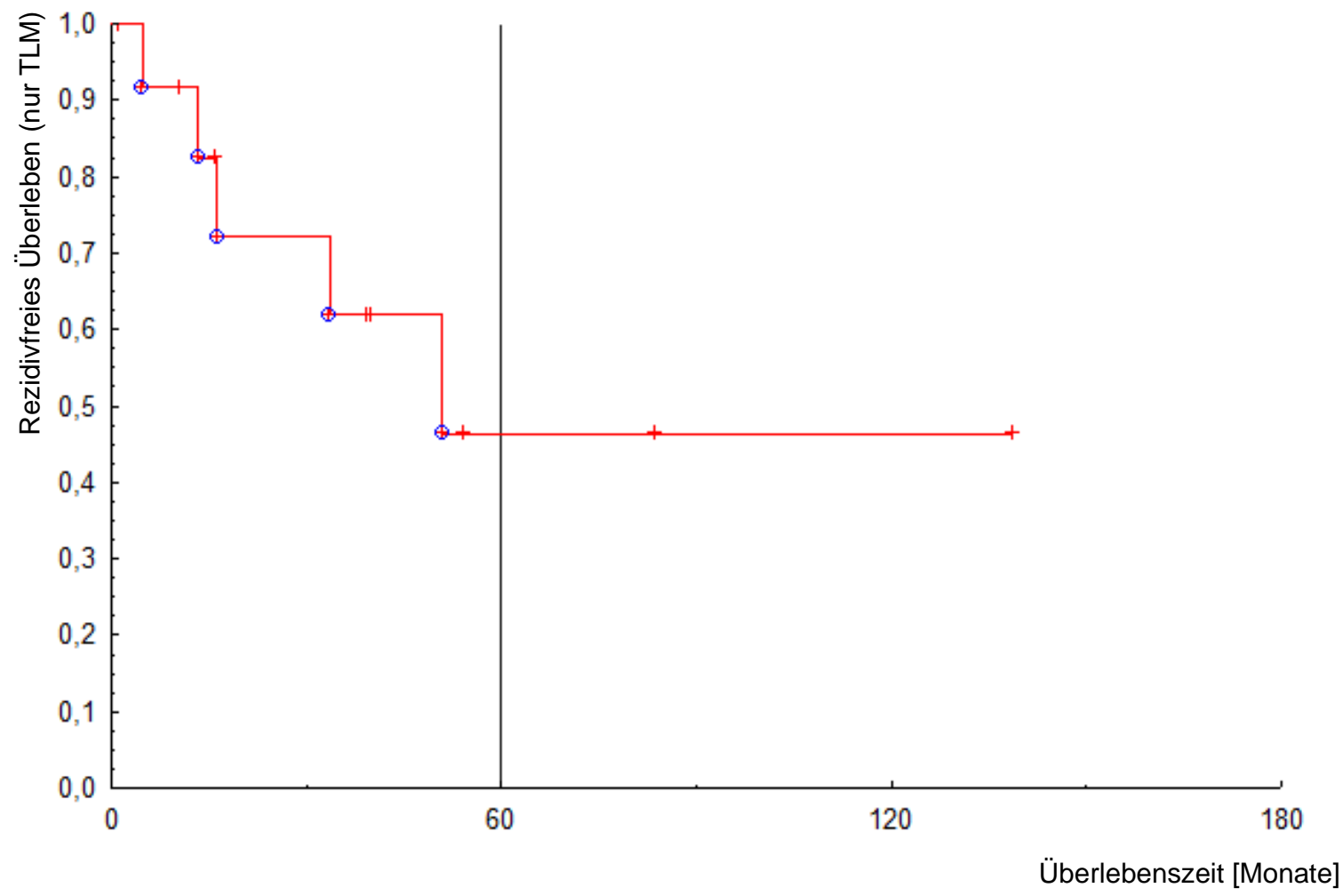

Abbildung 13: Kaplan-Meier-Schätzung für das rezidivfreie Überleben der primär lasermikrochirurgisch behandelten Patienten 


\subsection{Perioperatives Management, Komplikationen und funktionelle Ergebnisse}

\subsubsection{Perioperatives Management}

Eine elektive Tracheotomie erfolgte im Rahmen der transoralen lasermikrochirurgischen Tumoroperationen nicht. In einem Fall (8\% der 13 TLM-therapierten Patienten) wurde aufgrund einer operativ zu versorgenden Nachblutung am ersten Tag nach der Operation auch die Anlage eines Tracheostomas erforderlich. Das Tracheostoma wurde nach elf Tagen wieder verschlossen. Nach Lasermikrochirurgie wurde in $46 \%$ der Fälle $(n=5)$ eine nasogastrale Sonde gelegt, die nach durchschnittlich vier Tagen entfernt wurde. Folglich war bei allen TLM-behandelten Patienten im Verlauf eine reguläre orale Nahrungsaufnahme möglich.

Im Rahmen der primären Radiochemotherapie erhielt der eine auf diese Weise therapierte Patient eine Tracheotomie, die nach 59 Monaten wieder verschlossen wurde. Eine PEGErnährungssonde war in diesem Fall nicht erforderlich.

Die drei mittels Laryngektomie behandelten Patienten erhielten intraoperativ eine nasogastrale Sonde, die im Durchschnitt nach elf Tagen entfernt wurde, sodass die orale Nahrungsaufnahme wieder begonnen werden konnte. Das Tracheostoma behielten die Patienten, was in der Natur dieses Eingriffes liegt.

\subsubsection{Komplikationen}

Nach TLM ( $\mathrm{n}=13$ Patienten) kam es bei einem Patienten (7,7 \%) zu einer Nachblutung, die operativ versorgt werden musste. Hierbei war eine temporäre Tracheotomie erforderlich.

Die Laryngektomien verliefen ohne Komplikationen.

Im Rahmen einer beidseitigen Neck dissection (zehn Patienten erhielten im Rahmen der Primärtherapie eine Neck dissection) war schwellungsbedingt die Anlage eines Tracheostomas erforderlich. Bei diesem Patienten erfolgte die Neck dissection vier Wochen nach lasermikrochirurgischer Tumorresektion, ein unmittelbarer Zusammenhang zur TLM bestand folglich nicht. Bei diesem Patienten war zudem eine neurogene Schluckstörung dokumentiert (nach intraoperativer Pons-Blutung im Rahmen eines ohrchirurgischen Eingriffes), sodass nach der schwellungsbedingten Tracheotomie nach Neck dissection auch 
eine PEG-Ernährungssonde erforderlich wurde. Bis auf diesen Patienten war keine weitere PEG-Ernährungssonde im Rahmen der Primärtherapie erforderlich.

Ferner entwickelte sich bei zwei Patienten nach der Neck dissection ein Halshämatom, das operativ ausgeräumt werden musste.

\subsubsection{Funktionelle Ergebnisse}

Nach primär larynxerhaltender TLM ( $\mathrm{n}=13)$ war im Rahmen der Rezidivtherapie bei drei Patienten (23,1\%) eine Laryngektomie erforderlich. Die Rate des Larynxerhalts liegt folglich bei knapp $77 \%$.

Inklusive Rezidivtherapie wurden im Gesamtkollektiv sechs Kehlkopftotalentfernungen durchgeführt. Folglich konnte bei $65 \%$ der Patienten die Stimme erhalten werden.

Der primär radiochemotherapierte Patient entwickelte ein Rezidiv, dass bei zeitgleich diagnostizierter Fernmetastase im Rahmen eines palliativen Konzepts behandelt wurde. Eine Laryngektomie erfolgte nicht mehr. 


\section{Diskussion}

Die Therapiestrategie von Kopf-Hals-Tumoren wird in verschiedenen Kliniken und Ländern unterschiedlich gehandhabt. In den USA basiert die Behandlung häufig auf der primären Radiotherapie, wohingegen in Deutschland meist operative Therapiestrategien Anwendung finden (Wittekindt et al. 2012). Dies gilt auch für primär subglottisch gelegene Larynxkarzinome. An der Universitätsmedizin Göttingen etablierte Professor Steiner die transorale Lasermikrochirurgie in der Behandlung bösartiger Tumoren des gesamten oberen Aerodigestivtrakts (Steiner 1988; Steiner und Ambrosch 2000).

Mit etwa $2 \%$ ist die Subglottis als primäre Tumorlokalisation unter den Larynxkarzinomen selten (Ferlito und Rinaldo 2000). Folglich existieren nur wenig Studien mit onkologischen Daten verschiedener Therapiestrategien (Cassidy et al. 2012; Dahm et al. 1998; Garas und McGuirt 2006; Guedea et al. 1991; Paisley et al. 2002; Santoro et al. 2000; Shaha und Shah 1982; Su et al. 2003; Hata et al. 2013). Insbesondere existieren keine prospektiven randomisierten Studien, die eine Therapiestrategie gegen die andere vergleichen. Bei den existierenden Fallserien erschweren Unterschiede bezüglich der Einschlusskriterien, Kohortengröße, Verteilung der Tumorgrößen, Definition der superioren Begrenzung der Subglottis, Untersuchungszeiträume und statistischen Methoden den Vergleich der Studienergebnisse.

Das vorliegende Patientenkollektiv wurde mit 13 von 17 Patienten überwiegend transoral lasermikrochirurgisch behandelt. Davon erhielten fünf Patienten zusätzlich zur TLM eine Neck dissection und zwei weitere eine konkomitante Radiochemotherapie. Die chirurgische Dominanz in der Behandlung von Kopf-Hals-Karzinomen in Deutschland zeigt sich auch darin, dass nur ein Fall mittels primärer Radiochemotherapie behandelt wurde, drei Patienten erhielten eine totale Laryngektomie. Studien zu subglottischen Karzinomen in der Literatur beschreiben die Behandlungsstrategien der primären Radio(chemo)therapie (Cassidy et al. 2012; Guedea et al. 1991; Paisley et al. 2012; Hata et al. 2013) oder Laryngektomie (Santoro et al. 2000; Shaha und Shah 1982). Eine Übersicht zu den Publikationen gibt Tabelle 14. Die vorliegende Studie ist unseres Wissens die einzige, die ein vergleichsweise großes Kollektiv transoral lasermikrochirurgisch therapierter Patienten beschreibt. 
Das Auftreten von Rezidiven subglottischer Karzinome wird in der Literatur mit weit auseinanderliegenden Werten zwischen $14 \%$ und $81 \%$ beschrieben (Dahm et al. 1998; Santoro et al. 2000; Shaha und Shah 1982).

Nach Laryngektomie, primärer Radiotherapie oder einer Kombination aus beidem trat bei $14 \%(n=4)$ innerhalb eines Zeitraums von 3,7 Jahren ein Rezidiv auf (Dahm et al. 1998). beziehungsweise bei 52,9\% $(n=29)$ bei Santoro et al. (2000) bei einem Follow-up von fünf Jahren.

Im Vergleich dazu erkrankten $81 \%(n=13)$ innerhalb von fünf Jahren nach partieller oder totaler Laryngektomie an einem Rezidiv (Shaha und Shah 1982).

Demgegenüber steht eine Fünf-Jahres-Rezidivrate von $35 \%$ nach TLM in der vorliegenden Studie.

Ein Literaturvergleich zur vorliegenden Studie sollte unter Berücksichtigung der hohen Anzahl fortgeschrittener Tumorstadien erfolgen. In der vorliegenden Studie befand sich kein Patient zum Zeitpunkt der Diagnose im Stadium I. Zum Stadium II zählten vier Fälle (24 \%), drei Fälle wurden dem Stadium III (18 \%) zugeordnet und mit $59 \%$ zehn Fälle dem Stadium IV. Die Kaplan-Meier-Schätzungen ergaben ein Fünf-Jahres-Gesamtüberleben von 84,4 \% für das Gesamtkollektiv und 78,8 \% für ausschließlich primär lasermikrochirurgisch therapierte Patienten. Das krankheitsspezifische Überleben des vorliegenden Kollektivs lag bei 92,2 \% für die Gesamtkohorte beziehungsweise 90,0 \% für die Behandlung mit TLM. Ferner betrug das rezidivfreie Überleben 54,4 \% für das Gesamtkollektiv und 46,4 \% für die lasermikrochirurgisch behandelten Patienten.

Für ein mit der vorliegenden Studie vergleichbares Kollektiv in Bezug auf die Kohortengröße ( $\mathrm{n}=18)$ und Verteilung der T-Kategorien (67\% T3- oder T4-Karzinome), das mittels primärer Radiotherapie, Laryngektomie mit adjuvanter Radiatio oder neoadjuvanter Radiatio behandelt wurde, beschreiben Cassidy et al. (2012) ein Fünf-Jahres-Gesamtüberleben von $44 \%$ und ein krankheitsspezifisches Überleben von $66 \%$. Die Autoren weisen darauf hin, dass in ihrem Kollektiv Patienten an Komorbiditäten litten und deshalb nicht selten interkurrent verstorben sind. Hieraus resultiert die vergleichsweise niedrige Rate des Gesamtüberlebens (Cassidy et al. 2012).

Für ein ausschließlich radiotherapeutisch behandeltes Kollektiv von Paisley et al. (2002) lag das Fünf-Jahres-Gesamtüberleben für eine Kohorte der Größe von 43 Patienten bei 50,3 \%. Das krankheitsspezifische Überleben wird mit 52,0 \%, das rezidivfreie Überleben mit 66,9\% angegeben. Etwa 53 \% der Patienten hatten ein T1- oder T2-Karzinom, das mediane Followup lag bei vier Jahren. 
Nach Laryngektomie beziehungsweise partieller Laryngektomie wird von Shaha und Shah (1982) ein Fünf-Jahres rezidivfreies Überleben von 70,0 \% angegeben, ihr Kollektiv zählte 16 Fälle (T1 und T2: 19 \%, T3 und T4: 81 \%). In der vorliegenden Studie lag bei 76,5 \% der Patienten zum Diagnosezeitpunkt ein lokal fortgeschrittener Tumor (pT3: 23,5\%, pT4: $53 \%$ ) vor. Trotz der fortgeschrittenen Tumorstadien im vorliegenden Kollektiv liegen die Werte über den in der Literatur verfügbaren Studien. Ein Kollektiv mit im Vergleich größerem Anteil weniger fortgeschrittener Erkrankungen (68\% in den Stadien I und II) untersuchten Dahm et al. (1998) mit 28 Fällen mit primär subglottischen Plattenepithelkarzinomen. Die Patienten wurden radiotherapiert, total oder partiell laryngektomiert oder mit einer Kombination aus chirurgischer und radiotherapeutischer Strategie behandelt. Sie erreichten ein Fünf-Jahres-Gesamtüberleben von 57,7\%, ein krankheitsspezifisches Überleben von 46,2 \% und ein rezidivfreies Überleben von 38,5 \% nach fünf Jahren. Auch hier sind die Überlebensraten geringer als die der vorliegenden Studie, obwohl sich ein großer Anteil der Patienten im frühen Tumorstadium befand.

In der vorliegenden Analyse wurde nach fünf Jahren eine lokale Kontrollrate von 54,4 \% für das Gesamtkollektiv beziehungsweise 46,4 \% für ausschließlich primär lasermikrochirurgisch behandelte Patienten erzielt.

Nach primärer Radio(chemo)therapie hingegen erreichten Cassidy et al. (2012) eine FünfJahres lokale Kontrollrate von 83 \%. Paisley et al. (2002) erreichten eine Fünf-Jahres lokale Kontrollrate von 56 \%, Hata et al. (2012) erzielten eine Fünf-Jahres lokale Kontrollrate von $74 \%$. Sie stieg bei zusätzlicher Chemotherapie auf $82 \%$ an.

Eine Radiatio nach Salvage-Operation führte zu einer Fünf-Jahres lokalen Kontrolle von 81,4\% (Paisley et al. 2002).

Nach Laryngektomie erreichten Shaha und Shah (1982) in ihrer lediglich fünf Patienten zählenden Kohorte (T1: 1, T2: 2, T3: 1, T4: 1) eine lokale Kontrollrate von 100 \% nach fünf Jahren. Zu berücksichtigen ist jedoch, dass neben dem vergleichsweise geringen Umfang des Kollektivs zur Berechnung der lokalen Kontrollrate nicht auf die Kaplan-Meier-Methode zurückgegriffen wurde, sondern vielmehr Quotienten im Sinne von 5/5 (was $100 \%$ entspricht) angegeben worden sind. Aus diesen Gründen ist der Vergleich nur bedingt möglich.

Im Rahmen der chirurgischen Therapie erfolgte bei dem untersuchten Patientenkollektiv der vorliegenden Studie in etwa der Hälfte der Fälle (53,8 \%) zusätzlich zur TLM eine uni- oder bilaterale selektive Neck dissection. Bei sechs Patienten mit einem cN0-Status wurde auf eine 
elektive Neck dissection verzichtet. Diese zeigten in der Nachbeobachtung auch keine Lymphknotenmetastasen oder Spätmetastasen. Im Rahmen der Laryngektomie wurde in drei Fällen (100 \%) zusätzlich eine Ausräumung der Halslymphknoten durchgeführt. Insgesamt lag der Anteil der positiven Lymphknotenbefunde zum Zeitpunkt der Erstdiagnose bei 31,2 \% (fünf der 16 chirurgisch therapierten). Nach erfolgter Neck dissection entwickelten $20 \%$ (einer von den fünf Patienten) im weiteren Verlauf eine zervikale Metastase.

Zusammenfassend ist den vorliegenden Daten entsprechend der Wert einer elektiven Neck dissection fraglich. Auch Dahm et al. (1998) und Santoro et al. (2000) sehen in der Durchführung einer prophylaktischen Neck dissection keine Besserung der Überlebenszeiten.

Ein Ziel der TLM zur Behandlung von Malignomen des Larynx ist neben der onkologischen Kontrolle auch der Organ- und Funktionserhalt (Canis et al. 2013a; Canis et al. 2013b; Canis et al. 2014; Weiss et al. 2017). In der vorliegenden Studie war nach larynxerhaltender TLM ( $n=13)$ im Rahmen der Rezidivtherapie bei drei Patienten (23,1\%) eine Laryngektomie unausweichlich. Daraus resultiert eine Fünf-Jahres-Organerhaltungsrate von knapp 77 \%. Im Gegensatz dazu erreichte das hauptsächlich radiotherapeutisch behandelte Kollektiv von Cassidy et al. (2012) einen Organerhalt in zehn von 18 Fällen (56 \%). Ein ähnliches Ergebnis erzielten Paisley et al. (2012) ebenfalls nach Radiotherapie. Bei 24 von 43 Patienten konnte der Larynx erhalten werden (56\%). Angaben zur Nachbeobachtungszeit bezüglich des Organerhalts finden sich in den Studien nicht.

Da bei unseren Patienten zu 82,4\% keine Tracheotomie und zu 94,1\% keine PEG erforderlich war, ist in dieser Hinsicht von einem Funktionserhalt des Larynx auszugehen.

Eine geringe Komplikationsrate ist ein Ziel der Lasermikrochirurgie, die aufgrund des transoralen Zugangsweges mit einem geringen Trauma für nicht befallenes Gewebe einhergeht. Im vorliegenden Kollektiv kam es bei einem Patienten (7,7\%) nach TLM $(\mathrm{n}=13) \mathrm{zu}$ einer postoperativen Nachblutung und Schwellung. Diese musste operativ versorgt werden. Es erfolgte im Rahmen der Akutbehandlung eine temporäre Tracheotomie. Die Laryngektomien verliefen komplikationslos, wobei bei nur drei Fällen das Kollektiv für eine derartige Auswertung einen zu geringen Umfang aufweist. Im Zusammenhang mit der bilateralen Neck dissection (zehn Patienten erhielten eine Neck dissection im Rahmen der Primärtherapie) musste ein Patient schwellungsbedingt tracheotomiert werden. Die Neck dissection fand vier Wochen nach dem lasermikrochirurgischen Eingriff statt, sodass von einem Zusammenhang von Tracheotomie und TLM nicht auszugehen ist. Darüber hinaus 
entwickelte sich bei zwei Patienten (20\%) ein Halshämatom nach vorangegangener Neck dissection, welches operativ ausgeräumt werden musste.

Demgegenüber stehen Komplikationen, welche nach Radio(chemo)therapie auftraten. Als Folge einer Salvage-Operation nach vorausgegangener Strahlentherapie verstarb ein Patient (2\%) im Kollektiv von Paisley et al. (2002). Hämatologische und dermatologische Komplikationen nach Radio- oder Radiochemotherapie werden von Hata et al. (2012) beschrieben. Neben temporären Erythemen und Mukositiden, an denen $100 \%$ der Erkrankten postoperativ litten, traten zudem transiente Leukopenien auf (58 \%). Cassidy et al. (2012) berichten von lediglich einem Patienten, bei dem nach Radiatio Heiserkeit auftrat, die über sechs Monate persistierte. Bei 56 \% konnte die Stimme erhalten bleiben. Verglichen mit der TLM wurden im Rahmen der Rezidivtherapie im vorliegenden Kollektiv drei Kehlkopftotalentfernungen durchgeführt. Demnach konnte bei $77 \%$ der Patienten die Stimme erhalten werden.

Nach Laryngektomie trat im Kollektiv von Shaha und Shah ein Todesfall (6 \%) auf. Der Patient verstarb postoperativ nach Blutungen im oberen Gastrointestinaltrakt. Todesfälle als Folge von Komplikationen traten im vorliegenden Kollektiv nicht auf.

Darüber hinaus wurde nach Laryngektomie in Kombination mit Radiatio, alleiniger Laryngektomie oder alleiniger Radiatio eine ein- oder mehrmonatige Heiserkeit bei zehn Patienten (67\%) beschrieben (Garas und McGuirt 2006). In jeweils $17 \%$ der Fälle kam es zu Dysphagie beziehungsweise Dyspnoe. Eine Notfall-Tracheotomie musste in $20 \%$ der Fälle durchgeführt werden (Garas und McGuirt 2006).

Eine Tracheotomie wurde im Rahmen der transoralen Behandlung des Primärtumors nicht elektiv angelegt. Wie bereits beschrieben, war bei einem der 13 untersuchten Patienten des vorliegenden Kollektivs nach Laserresektion aufgrund einer postoperativen Schwellung die temporäre Anlage eines Tracheostomas erforderlich, das nach elf Tagen wieder verschlossen wurde. Ferner erhielt ein Patient des untersuchten Kollektivs im Rahmen der primären Radiotherapie ein Tracheostoma, welches nach 59 Monaten verschlossen wurde.

Drei mittels Laryngektomie behandelte Patienten (100\%) erhielten intraoperativ eine nasogastrale Sonde, die im Durchschnitt nach elf Tagen entfernt wurde. Im Rahmen der TLM wurde in $46 \%$ der Fälle $(n=5)$ eine nasogastrale Sonde gelegt, die nach durchschnittlich vier Tagen entfernt wurde. Im Verlauf war also bei allen primär lasermikrochirurgisch behandelten Patienten eine reguläre orale Nahrungsaufnahme möglich. Die TLM ermöglicht folglich wie auch die Laryngektomie eine schnelle Wiederaufnahme einer regulären oralen Ernährung. 
In der Literatur zur Behandlung subglottischer Karzinome finden sich zum perioperativen Management, insbesondere zur Notwendigkeit einer Tracheotomie, wenig Angaben. In einem sechs Patienten umfassenden Kollektiv primär radiotherapeutisch behandelter Patienten benötigten zwei Patienten (33\%) bei zunehmendem Stridor eine temporäre Tracheotomie (Guedea et al. 1991). In einem Fall wurde das Tracheostoma nach einem Monat, im anderen Fall den Angaben nach während der zweiten Behandlungshälfte verschlossen, wobei dies zeitlich nicht näher benannt wird. In einem 43 Patienten umfassenden Kollektiv primär radiotherapierter Patienten war keine Tracheotomie erforderlich (Paisley et al. 2002). Abschließend muss erwähnt werden, dass nach Laryngektomie aufgrund der Trennung von Luft- und Speiseweg die Atmung obligat über ein Tracheostoma erfolgt. Hierin liegt ein Vorteil der organerhaltenden Therapieverfahren, der Radiotherapie sowie der transoralen Lasermikrochirurgie. 


\begin{tabular}{|c|c|c|c|c|}
\hline 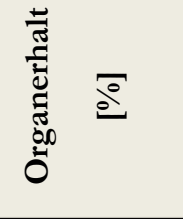 & 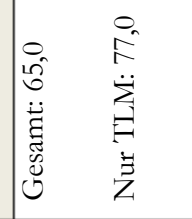 & 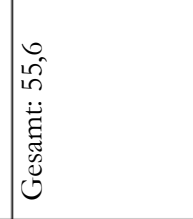 & 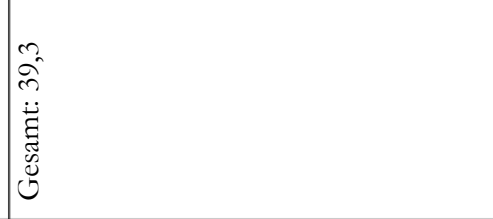 & 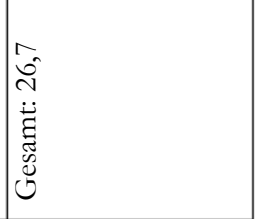 \\
\hline 聯 & 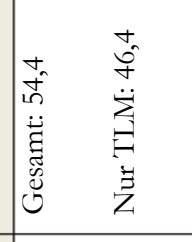 & 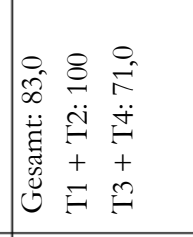 & & 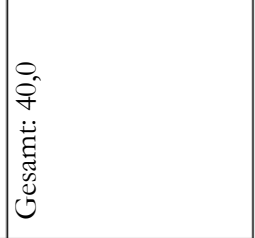 \\
\hline 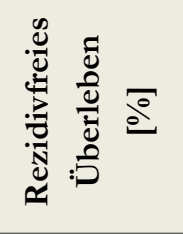 & 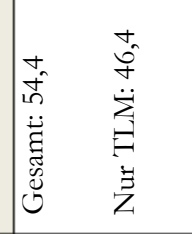 & & 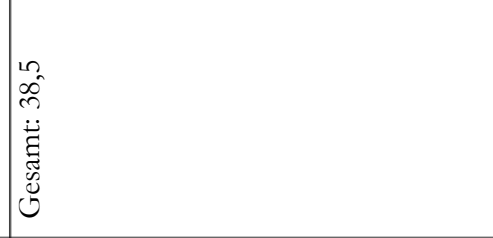 & \\
\hline 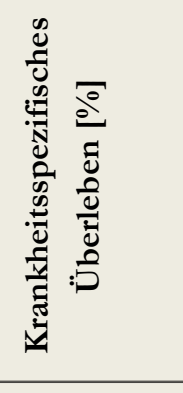 & 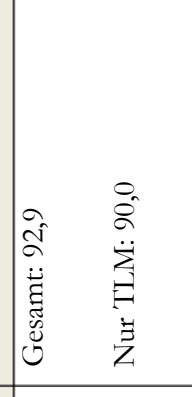 & 家 & 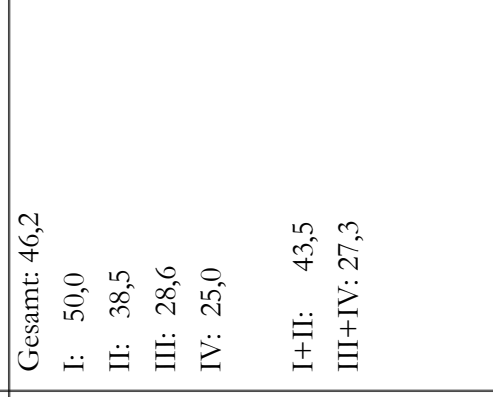 & 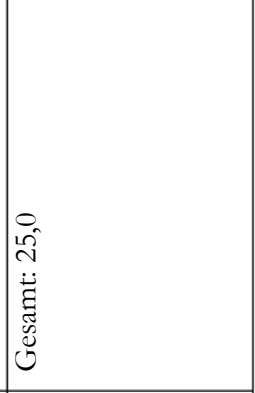 \\
\hline 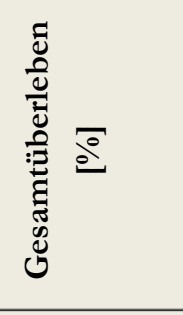 & 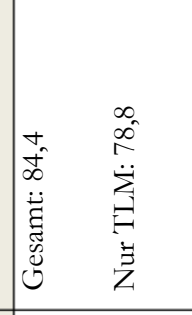 & $\mid \begin{array}{l}0 \\
f \\
f\end{array}$ & 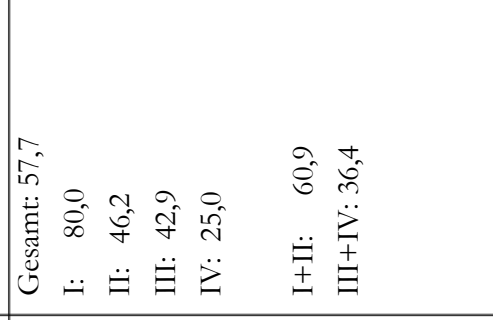 & 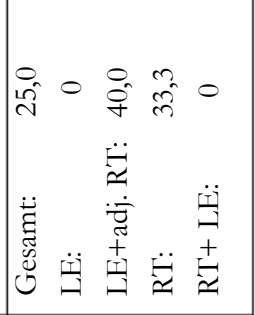 \\
\hline 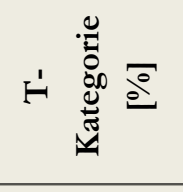 & 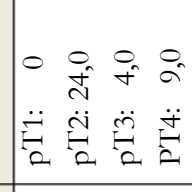 & 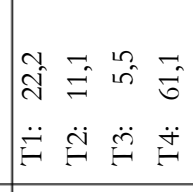 & 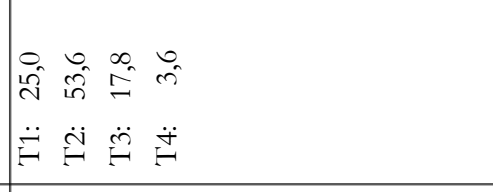 & 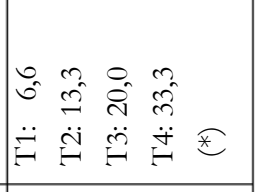 \\
\hline 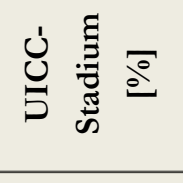 & 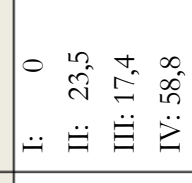 & & 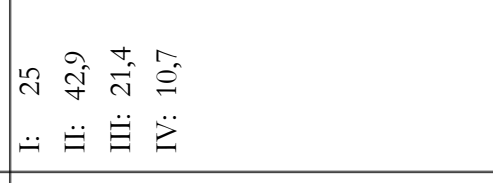 & \\
\hline 今े & 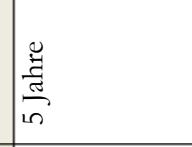 & 总 & 尊 & 爰 \\
\hline 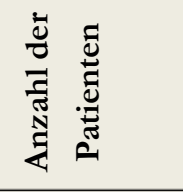 & $\nexists$ & $\stackrel{\infty}{-}$ & $\infty$ & $\cong$ \\
\hline . & 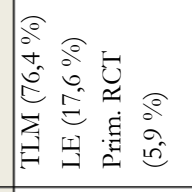 & 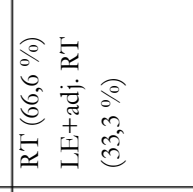 & 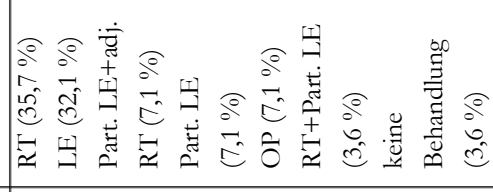 & 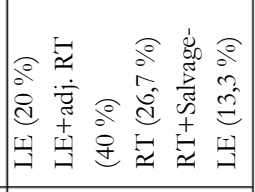 \\
\hline 窇 & 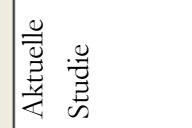 & 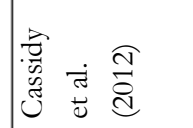 & 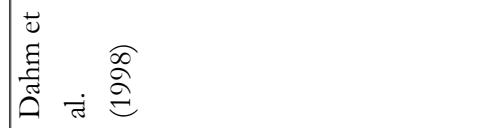 & 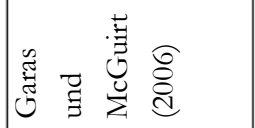 \\
\hline
\end{tabular}




\begin{tabular}{|c|c|c|c|c|c|c|}
\hline 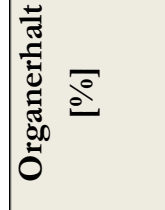 & 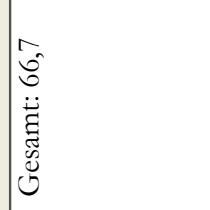 & 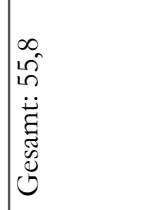 & 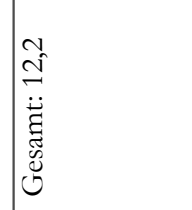 & 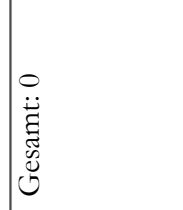 & 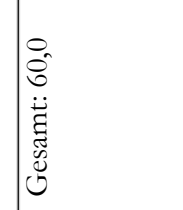 & 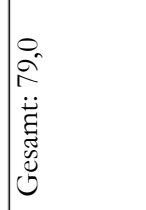 \\
\hline 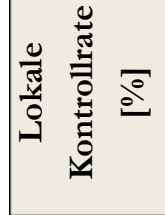 & 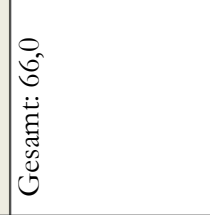 & 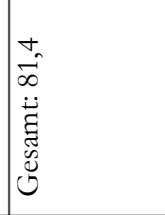 & 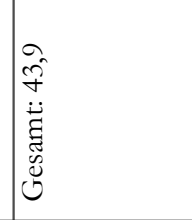 & & $\begin{array}{l}\frac{\pi}{2} \\
\text { 童 } \\
\text { in }\end{array}$ & 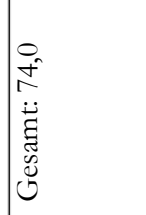 \\
\hline 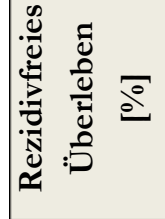 & & 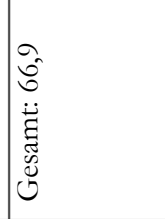 & & 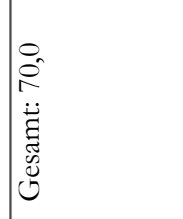 & 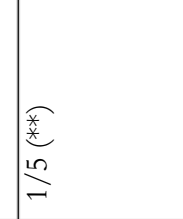 & 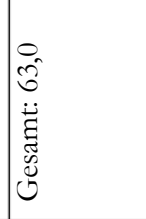 \\
\hline 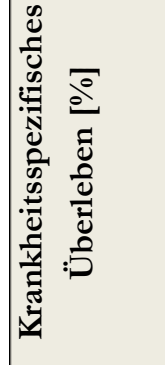 & & 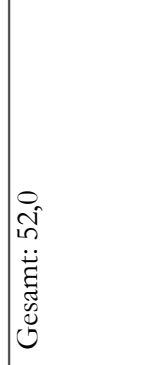 & 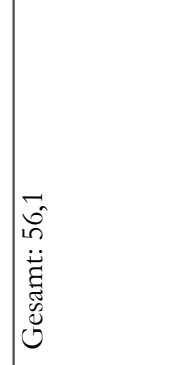 & & & 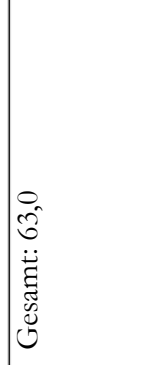 \\
\hline 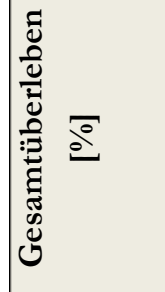 & & 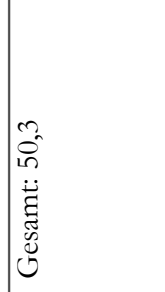 & & & & 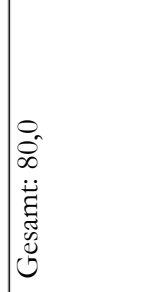 \\
\hline 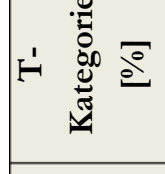 & 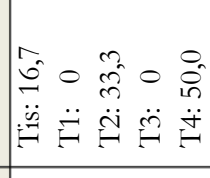 & 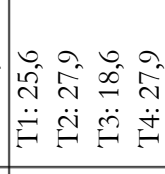 & 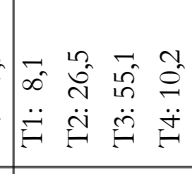 & 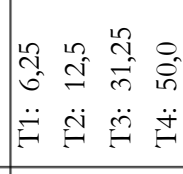 & 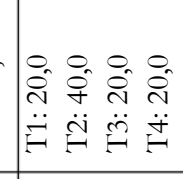 & 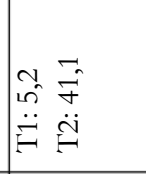 \\
\hline 远 & & & & & & \\
\hline 密 & $\frac{\frac{\mathrm{g}}{\mathrm{s}}}{\mathrm{s}}$ & 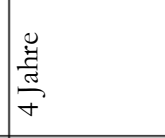 & 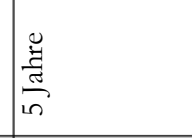 & 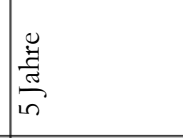 & 惫 & 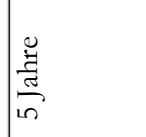 \\
\hline 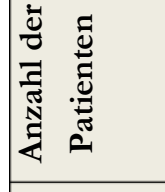 & 0 & 7 & f & $\because$ & in & 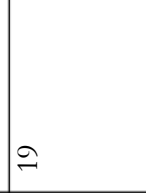 \\
\hline |. & 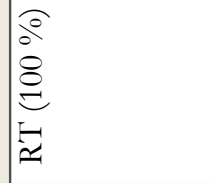 & 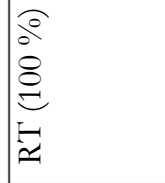 & 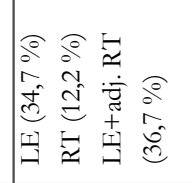 & 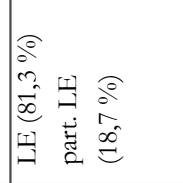 & 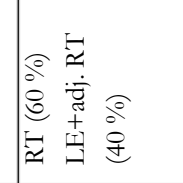 & 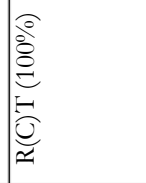 \\
\hline : & 㺃 & 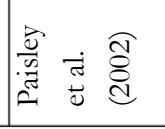 & 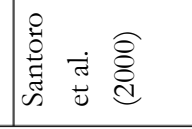 & 烝 & 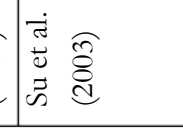 & 的离 \\
\hline
\end{tabular}




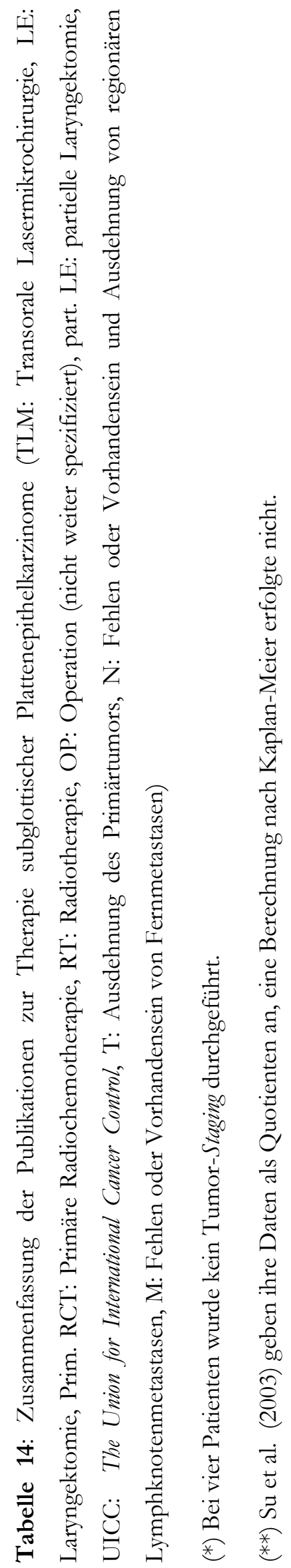




\subsection{Limitationen der Studie}

Eine Limitation der vorliegenden Studie ist ihr retrospektives Design. Unmittelbare Vergleiche unterschiedlicher Therapiestrategien waren nicht möglich, da es an der Universitätsmedizin Göttingen keine vergleichbar großen Patientenkollektive gab. Auch ermöglichte die Auswertung keine Beurteilung der Stimmbildungsfunktion des Larynx.

Zudem stellt die Uneinigkeit bezüglich der superioren Begrenzung der Subglottis eine Schwierigkeit beim Vergleich der Studien dar. Ferner erschweren im Literaturvergleich unterschiedliche Einschlusskriterien und statistische Methoden den Vergleich onkologischer Ergebnisse.

\subsection{Stärken der Studie}

Zu den Stärken der Studie zählt die Datenqualität aufgrund einer umfangreichen Dokumentation der Patientenfälle aus der Klinik für Hals-Nasen-Ohrenheilkunde der Universitätsmedizin Göttingen sowie die Darstellung von Behandlungsergebnissen aus einer Klinik mit weltweit beachteter Expertise in der transoralen Lasermikrochirurgie zur Behandlung von Kopf-Hals-Malignomen. Anhand von detaillierten Operationsberichten ließ sich die chirurgische Therapie nachvollziehen und die Tumorlokalisationen waren genau zu bestimmen. Ferner ist auch die Kohortengröße eine Stärke, wobei berücksichtigt werden muss, dass es sich bei primär subglottischen Malignomen um eine seltene Erkrankung handelt. Im Durchschnitt umfassten die bislang publizierten Studien 22 Patienten. Die vorliegende Studie liegt mit 17 Patienten leicht darunter, ist aber die einzige, die Ergebnisse zur transoralen Lasermikrochirurgie in der Behandlung subglottischer Tumoren darstellt. 


\section{Zusammenfassung und Schlussfolgerung}

Primär subglottisch auftretende Plattenepithelkarzinome stellen unter Larynxmalignomen eine seltene Tumorentität dar. Begünstigt wird ihr Auftreten durch Alkohol- und Nikotinkonsum, auch eine genetische Disposition ist von Bedeutung. In frühen Tumorstadien sind subglottische Malignome asymptomatisch. Zum Zeitpunkt der Diagnose liegt folglich meist eine fortgeschrittene Erkrankung vor. Aus diesem Grund hat ein subglottsiches Karzinom in der Literatur eine schlechte Prognose. Zu den Therapieregimen zählen neben der primären Radio(chemo)therapie die konventionell offen chirurgische partielle oder totale Laryngektomie. Professor Steiner aus Göttingen therapierte erstmalig laryngeale Malignome mithilfe der minimalinvasiven transoralen Lasermikrochirurgie, nachdem die Methode zuvor in den Vereinigten Staaten für benigne laryngeale Läsionen Anwendung fand. Beginnend mit glottischen Tumoren weitete er den Einsatz der transoralen Lasermikrochirurgie auf den gesamten oberen Aerodigestivtrakt aus.

Ziel der vorliegenden Studie war es, die onkologischen und funktionellen Ergebnisse sowie das perioperative Management und die Komplikationen der transoralen Lasermikrochirurgie in der Behandlung subglottischer Karzinome zu evaluieren.

Das vorliegende Kollektiv bestand aus 17 Patienten, die zwischen 1992 und 2013 in der Klinik für Hals-Nasen-Ohrenheilkunde der Universitätsmedizin Göttingen in kurativer Intention therapiert wurden. Zum Diagnosezeitpunkt lag bei 76,2\% ein fortgeschrittenes Tumorstadium vor. In den meisten Fällen (76,4\%) wurde organerhaltend, primär lasermikrochirurgisch behandelt. Ebenfalls mit dem Ziel des Organerhalts erhielten 5,9\% eine primäre Radio(chemo)therapie, 17,6 \% wurden mittels totaler Laryngektomie behandelt. Die mittlere Nachsorgeperiode betrug 70 Monate. Das Fünf-Jahres-Gesamtüberleben nach transoraler Lasermikrochirurgie lag bei 78,8\%, das krankheitsspezifische Überleben bei 90,0 \% das rezidivfreie Überleben und die lokale Kontrollrate lagen jeweils bei 46,4 \%. Der Larynxerhalt nach transoraler Lasermikrochirurgie lag bei 77,0 \%.

Studien zu onkologischen Ergebnissen dieser seltenen Tumorentität sind rar. Die Therapie subglottischer Malignome mittels transoraler Lasermikrochirurgie unter Berücksichtigung der bislang veröffentlichten Untersuchungen sind ein Alleinstellungsmerkmal der vorliegenden Studie. 
Unter Berücksichtigung der bedingt möglichen Vergleichbarkeit onkologischer Ergebnisse retrospektiver Studien erzielte die transorale Lasermikrochirurgie, gegebenenfalls in Kombination mit einer Neck dissection und/oder adjuvanten Radio(chemo)therapie, in der Behandlung primär subglottisch gelegener Karzinome mindestens vergleichbare onkologische Ergebnisse zur Laryngektomie oder primären Radio(chemo)therapie. Sie kann folglich in spezialisierten Zentren mit ausreichender Expertise in der transoralen Lasermikrochirurgie mit dem Ziel des Organerhalts neben der primären Radiochemotherapie eine Behandlungsoption für subglottische Karzinome darstellen. 


\section{Literaturverzeichnis}

Ambrosch P (2007): The role of laser microsurgery in the treatment of laryngeal cancer.

Otolaryngol Head Neck Surg $\underline{15}, 82-88$

Bernier J, Domenge C, Ozsahin M, Matuszewska K, Lefebre JL, Greiner RH, Giralt J, Maingon P, Rolland F, Bolla M (2004): Postoperative irradiation with or without concomitant chemotherapy for locally advanced head and neck cancer. N Engl J Med $\underline{350}, 1945-1952$

Canis M, Ihler F, Martin A, Wolff HA, Matthias C, Steiner W (2013a): Organ preservation in T4a laryngeal cancer: is transoral laser microsurgery an option? Eur Arch Otorhinolaryngol 270, 2719-2727

Canis M, Martin A, Ihler F, Wolff HA, Kron M, Matthias C, Steiner W (2013b): Transoral laser microsurgery in treatment of pT2 and pT3 glottic laryngeal squamous cell carcinoma - results of 391 patients. Head Neck $\underline{36}$, 889-895

Canis M, Ihler F, Martin A, Matthias C, Steiner W (2014): Transoral laser microsurgery for T1a glottic cancer: review of 404 cases. Head Neck $\underline{37}, 889-895$

Cassidy R, Morris CG, Kirwan JM, Amdur RJ, Mendenhall WM (2012): Radiation therapy for squamous cell carcinoma of the subglottic larynx. J Radiat Oncol 1, 333-336

Cattaruzza MS, Maisonneuve P, Boyle P (1996): Epidemiology of laryngeal cancer. Eur J Cancer B Oral Oncol $\underline{32 B}, 293-305$

Cooper JS, Pajak TF, Forastiere AA, Jacobs J, Campbell BH, Saxman SB, Kish JA, Kim HE, Cmelak AJ, Rotman M (2004): Postoperative concurrent radiotherapy and chemotherapy for high risk squamous cell carcinoma of the head and neck. N Engl J Med $\underline{350}, 1937-1944$

Cornelius CP, Andratschke M, Leunig A, Hilscher C: Neck Dissection. In: Mast G (Hrsg.): MANUAL Kopf-Hals-Malignome. W. Zuckschwerdt Verlag, München 2009, 52-60

Dahm JD, Sessions DG, Paniello RC, Harvey J (1998): Primary subglottic cancer. Laryngoscope 108, 741-746 
Delank KW, Stoll W (2000): Moderne Diagnostik und Therapie bei Kehlkopftumoren: ein Überblick. Dtsch. Med. Wschr. 125, 1169-1172

Dellian M: Malignome des Kehlkopfes. In: Mast G (Hrsg.): MANUAL Kopf-HalsMalignome. W. Zuckschwerdt Verlag, München 2009, 49-51

Dempke W: Lehrbuch Hämato-Onkologie. 1. Auflage; Verlag Hans Huber, Bern 2006

DKG (2019): Diagnostik, Therapie und Nachsorge des Larynxkarzinoms. S3-Leitlinie der Deutschen Krebsgesellschaft e.V. und der Deutschen Krebshilfe. http://www.leitlinienprogramm-onkologie.de/leitlinien/larynxkarzinom/; abgerufen am 29.02.2020

Edge SB, Compton CC (2010): The American Joint Committee on Cancer: the 7th edition of the AJCC cancer staging manual and the future of TNM. Ann Surg Oncol $\underline{17}, 1471$ 1474

Ferlito A, Rinaldo A (2000): The pathology and management of subglottic cancer. Eur Arch Otorhinolaryngol 257, 168-173

Fritsch H, Kühnel W: Taschenatlas Anatomie. 10. Auflage; Georg Thieme Verlag, Stuttgart 2009

Garas J, McGuirt WF (2006): Squamous cell carcinoma of the subglottis. Am J Otolaryngol $\underline{27}, 1-4$

Genden EM, Ferlito A, Silver CE, Jacobson AS, Werner JA, Suárez C, Leemans CR, Bradley PJ, Rinaldo A (2007): Evolution of the management of laryngeal cancer. Oral Oncol $\underline{43}$, 431-439

Guedea F, Parsons JT, Mendenhall WM, Million RR, Stringer SP, Cassisi NJ (1991): Primary subglottic cancer: results of radical radiation therapy. Int J Radiat Oncol Biol Phys $\underline{21}$, $1607-1611$

Haas I (2000): Tumornachsorge: Ziele, Ergebnisse und Konzepte. Laryngo-Rhino-Otol 79, $667-668$

Harrison DFN (1971): The pathology and management of subglottic cancer. Ann Otol Rhinol Laryngol 우, 6-12 
Hata M, Taguchi T, Koike I, Nishimura G, Takahashi M, Komatsu M, Sano D, Odagiri K, Minagawa Y, Inoue T (2013): Efficacy and toxicity of (chemo)radiotherapy for primary subglottic cancer. Strahlenther Onkol 189, 26-32

Hermanek P, Scheibe O, Spiessl B, Wagner G: TNM-Klassifikation maligner Tumoren. 4. Auflage; Springer, Berlin 1992

Hoffman HT, Karnell LH, Funk GF, Robinson RA, Menck HR (1998): The national cancer data base report on cancer of the head and neck. Arch Otolaryngol Head Neck Surg $\underline{124}$, 951-962

Iro H, Waldfahrer F: Larynx und Trachea. In: Probst R, Grevers G, Iro H (Hrsg.): HalsNasen-Ohrenheilkunde. Georg Thieme Verlag, Stuttgart 2008, 305-348

Kaplan EL, Meier P (1958): Nonparametric estimation from incomplete observations. J Am Stat Assoc $\underline{53}, 457-481$

Karamanou M, Markatos K, Lymperi M, Agapitos E, Androutsos G (2017): A historical overview of laryngeal carcinoma and the first total laryngectomies. J BUON 22, 807-811

Lenarz T, Boenninghaus HG: Hals-Nasen-Ohrenheilkunde. 14. Auflage; Springer, Berlin 2012

Levy A, Blanchard P, Temam S, Maison MM, Janot F, Mirghani H, Bidault F, Guigay J, Lusinchi A, Bourhis J (2014): Squamous cell carcinoma of the larynx with subglottic extension: is larynx preservation possible? Strahlenther Onkol 190, 654-660

Moll KJ, Moll M: Anatomie. 18. Auflage; Elsevier Urban \& Fischer, München 2006

Mozet C, Dietz A (2010): Malignome des Larynx. Laryngo-Rhino-Otol 무, 295-315

Oehler C (2014): Spätfolgen der Strahlentherapie. SZO $\underline{4}, 10-16$

Paisley S, Warde PR, O’Sullivan B, Waldron J, Gullane PJ, Payne D, Liu FF, Bayley A, Ringash J, Cummings BJ (2002): Results of radiotherapy for primary subglottic squamous cell carcinoma. Int J Radiat Oncol Biol Phys $\underline{52}$, 1245-1250 
Papachristofilou A, Oduncu FS, Zimmermann F, Mast G: Allgemeine Grundsätze der Strahlentherapie. In: Mast G (Hrsg.): MANUAL Kopf-Hals-Malignome. W. Zuckschwerdt Verlag, München 2009, 167-171

Pignon JP, Bourhis J, Domenge C, Designé L (2000): Chemotherapy added to locoregional treatment for head and neck squamous cell carcinoma: three metaanalyses of updated individual data. Lancet $\underline{355}, 949-955$

Pigorsch S, Röper B, Wypior H, Zimmermann F: Allgemeine Grundsätze der Strahlentherapie. In: Mast G (Hrsg.): MANUAL Kopf-Hals-Malignome. W. Zuckschwerdt Verlag, München 2009, 70-72

Ramroth H, Dietz A, Becher H (2006): Rauchen und Alkohol sind Hauptrisikofaktoren für Kehlkopfkrebs. Dtsch Arztebl 103, 1078-1083

Rudert $\mathrm{H}$, Werner JA: $\mathrm{CO}_{2}$ laser surgery of benign and malignant lesions of the oral cavity, pharynx and larynx. Endo Press, Tuttlingen 2011

Santoro R, Turelli M, Polli G (2000): Primary carcinoma of the subglottic larynx. Eur Arch Otorhinolaryngol 257, 548-551

Sapundzhiev NR, Dünne AA, Ramaswamy A, Sitter H, Davis RK, Werner JA (2005): Lymph node metastasis in an animal model: effect of piecemeal laser surgical resection. Lasers Surg Med $\underline{36}$, 371-376

Schmoll HJ, Dunst J, Becker A, Flieg WE (1997): Simultane Radiochemotherapie. Dtsch Arztebl 94, A-3277-3280

Schuler K, Winter W, Betz CS, Leunig A: Tumorresektion. In: Mast G (Hrsg.): MANUAL Kopf-Hals-Malignome. W. Zuckschwerdt Verlag, München 2009, 43-48

Schwab W (1955): Über morphologische und funktionelle Veränderungen am Atmungstrakt nach Laryngektomie. Arch Ohren Nasen Kehlkopfheilkd 166, 444-475

Schwab W, zum Winkel K: Möglichkeiten der Strahlentherapie in der Hals-NasenOhrenheilkunde. In: Becker W, Boenninghaus HG, Leicher H, Mittermaier R, Naumann HH, Theisting G (Hrsg.): Aktuelle Oto-Rhino-Laryngologie. Georg Thieme Verlag, Stuttgart 1975, 57-67

Shaha AR, Shah JP (1982): Carcinoma of the subglottic larynx. Am J Surg 144, 456- 458 
Shin JY, Truong MT (2015): Racial disparities in laryngeal cancer treatment and outcome: a population-based analysis of 24,069 patients. Laryngoscope $\underline{125}, 1667-1674$

Siefert A, Pollinger B, Papachristofilou A, Zimmermann F, Panzer M: Kombinierte Radiochemotherapie. In: Mast G (Hrsg.): MANUAL Kopf-Hals-Malignome. W. Zuckschwerdt Verlag, München 2009, 84-87

Sobin LH, Compton CC (2010): TNM seventh edition: what's new, what's changed: communication from the International Union Against Cancer and the American Joint Committee on Cancer. Cancer 116, 5336-5339

Sobotta Atlas der Anatomie. Hrsg. v. Paulsen F, Waschke J, 24. Auflage; Urban und Fischer in Elsevier, München 2017

Steiner W (1988): Experience in endoscopic laser surgery of malignant tumours of the upper aerodigestive tract. Adv Otorhinolaryngol $\underline{39}$, 135-144

Steiner W, Ambrosch P: Endoscopic laser surgery of the upper aerodigestive tract: with special emphasis on cancer surgery. Thieme, Germany and New York 2000

Steiner W, Howard D, Haughey B, Bernal-Sprekelsen M: Transoral laser microsurgery for cancer of the upper aerodigestive tract. Endo Press, Tuttlingen 2013

Steuer CE, El-Deiry M, Parks JR, Higgins KA, Saba NF (2017): An update on larynx cancer. CA Cancer J Clin $\underline{67}, 31-50$

Strong MS, Jako GJ (1972): Laser surgery in the larynx. Early clinical experience with continous $\mathrm{CO}_{2}$ laser. Ann Otol Rhinol Laryngol $\underline{81}, 791-798$

Su WF, Jen YM, Nieh S (2003): Multifocal carcinoma of the larynx presenting as subglottic carcinoma. Eur Arch Otorhinolaryngol 260, 211-215

Van Kampen M: Strahlentherapie. In: Hübner J (Hrsg.): Onkologie interdisziplinär. Schattauer, Stuttgart 2014, 43-46

Weiss BG, Ihler F, Pilavakis Y, Wolff HA, Canis M, Welz C, Steiner W (2017): Transoral laser microsurgery for Tb1 glottic cancer: review of 51 cases. Eur Arch Otorhinolaryngol 274, 1997-2004 
Werner JA: Durchführung der Tumortherapie. In: Zenner HP (Hrsg.): Praktische Therapie von HNO-Krankheiten. Schattauer, Stuttgart 2008, 36-37

Werner JA, Lippert BM, Schünke M, Rudert H (1995): Tierexperimentelle Untersuchungen zur Laserwirkung auf Lymphgefäße. Laryngo-Rhino-Otol $\underline{74}$, 748-755

Wiegand S, Wiemers C, Murthum T, Zimmermann AP, Bette M, Mandic R, Werner JA (2013): Risk of lymph node metastases after en bloc cold steel, en bloc laser-, and piecemeal laser surgical resection of auricular VX2-carcinoma. Lasers Med Sci 28, $1137-1141$

Wittekindt C, Wagner S, Mayer CS, Klußmann JP (2012): Grundlagen der Tumorentstehung und die Bedeutung Humaner Papillomaviren (HPV) bei Kopf-HalsKarzinomen. Laryngo-Rhino-Otol 1ㅗ, 1-26

Young N, Mangus BD, Bumpous JM (2008): Salvage laryngectomy for failed Conservative treatment of laryngeal cancer. Laryngoscope 118, 1561-1568

ZfKD (Zentrum für Krebsregisterdaten): Bericht zum Krebsgeschehen in Deutschland 2016. Robert Koch-Institut, Berlin 2016

Zimmermann F, Matthias C: Malignome des Kehlkopfes. In: Mast G (Hrsg.): MANUAL Kopf-Hals-Malignome. W. Zuckschwerdt Verlag, München 2009, 272-280 


\section{Danksagung}

Mein besonderer Dank gilt meinem Doktorvater, Herrn Prof. Dr. Ihler, für die Überlassung des Themas, für die Anregungen und das mir entgegengebrachte Vertrauen. Trotz des Wechsels nach München haben Sie mir stets zuverlässig rasche Rückmeldungen gegeben. Herzlichen Dank!

Meinem Betreuer, Herrn PD Dr. Weiß, bin ich ebenfalls zu großem Dank verpflichtet - für die intensive Einarbeitung in das Thema, die Unterstützung bei der statistischen Analyse und der geduldigen Beantwortung meiner Fragen zu jeder Zeit. Die Zusammenarbeit mit dir hat mir gezeigt, was es heißt, wissenschaftlich zu arbeiten.

Frau Bruhse möchte ich für die Unterstützung bei der Aktensuche und für die Mitbenutzung ihres Büros danken.

Außerdem möchte ich meiner Mitpromovendin Marie Kruizenga danken - für die Motivation, den kollegialen Austausch, die unzähligen gemeinsamen Stunden in der SUB und die Tage in Hamburg. Ohne dich wäre die Entstehung der Dissertation nur halb so lustig gewesen. 